\title{
THE EXISTENCE OF NONTRIVIAL CRITICAL POINT FOR A CLASS OF STRONGLY INDEFINITE ASYMPTOTICALLY QUADRATIC FUNCTIONAL WITHOUT COMPACTNESS
}

\author{
Guanggang Liu — ShaOyun Shi* — Yucheng Wei
}

\begin{abstract}
In this paper, we show the existence of nontrivial critical point for a class of strongly indefinite asymptotically quadratic functional without compactness, by using the technique of penalized functionals and an infinite dimensional Morse theory developed by Kryszewski and Szulkin. Two applications are given to Hamiltonian systems and elliptic systems.
\end{abstract}

\section{Introduction}

Let $E$ be a real Hilbert space with an inner product $\langle\cdot, \cdot\rangle$ and norm $\|\cdot\|$. Consider the functional of the following form

$$
\Phi(x)=\frac{1}{2}\left\langle L_{\infty} x, x\right\rangle-\varphi(x),
$$

where $L_{\infty}: E \rightarrow E$ is a bounded linear selfadjoint Fredholm operator of index 0 , $\nabla \varphi(0)=0, \nabla \varphi$ is a compact mapping and $\nabla \varphi(x) \rightarrow 0$ as $\|x\| \rightarrow \infty$. Moreover, we suppose that $0 \in \sigma\left(L_{\infty}\right)$ and $\Phi \in C^{2}(E, \mathbb{R})$ is a strong indefinite functional,

2010 Mathematics Subject Classification. Primary 58E05; Secondary 34C25, 58F05, 35J65.

Key words and phrases. Strongly indefinite functional, filtration, Morse index, critical group, Hamiltonian system, elliptic system.

This work was supported by NSFC grant (11371166) and National 973 project of China (2012CB821200), SRFDP grant (20060183017).

* The corresponding author. 
i.e. $\Phi$ is unbounded from below and from above on any subspace of finite codimension. Obviously, $\Phi$ is an asymptotically quadratic functional with a trivial critical point 0 .

The purpose of this paper is to find the nontrivial critical point of $\Phi$. There are two difficulties in treating this problem. On the one hand, since $\Phi$ is a strong indefinite functional, it is well known that the Morse index of any critical point of $\Phi$ must necessarily be infinite, therefore we can not expect to obtain any useful information from the usual Morse theory. On the other hand, since $0 \in \sigma\left(L_{\infty}\right)$, the global compactness of $\Phi$ may be lost.

To overcome the difficulties caused by the strongly indefinite property of functional, some new techniques were developed. In 1997, Kryszewski and Szulkin[12] developed an infinite dimensional Morse theory, which was applied to the asymptotically linear Hamiltonian systems, wave equations and elliptic systems. By developing a method to compute the new cohomology critical groups both at zero and at infinity precisely, A. Szulkin, W. M. Zou[23] and W. M. Zou[25] obtained the existence of (multiple) nontrivial solutions for asymptotically linear Hamiltonian systems, beam equations and noncooperative elliptic systems.

In 1997, Abbondandolo [1] developed another Morse theory for strongly indefinite functionals, which was applied to study the existence of nontrivial periodic solutions for Hamiltonian systems. In [17], a similar result was obtained for an asymptotically linear non-cooperative elliptic system by Abbondandolo's theory.

In [8], a new Morse index theory for strongly indefinite functionals was developed via Galerkin approximation. This method was applied to the asymptotically linear Hamiltonian systems [9], noncooperative elliptic systems [10], wave equation and beam equations [24], respectively.

To overcome the difficulties caused by resonance, some kinds of conditions were imposed to ensure the compactness of functional, for example, LandsmanLazer type condition [13]. In [15], A. Masiello and L. Pisani considered a bounded resonance problem for semilinear elliptic equations. Since the assumptions that imposed to the nonlinearities can not ensure the compactness of functional, they used the technique of penalized functional. In [21], Su and Liu extended the above result to the nonautonomous case with resonance both at zero and at infinity. Moreover, they considered the multiplicity and sign-changing properties of nontrivial solutions via the classical Morse theory.

In this paper, we consider the general bounded resonance strongly indefinite variational problem with the nonlinearity near infinity similar to that in [15] and [21]. By using the infinite dimensional Morse theory developed by Kryszewski and Szulkin and the technique of penalized functional, we obtain the existence of nontrivial critical point for functional (1.1). As applications, we 
studied the existence of nontrivial solutions for Hamiltonian system and a class of strongly indefinite elliptic system, respectively.

The paper is organized as follows. In Section 2, we introduce the infinite dimensional Morse theory established by Kryszewski and Szulkin as preliminaries. The main result and its proof will be given in Section 3. In Sections 4 and 5, some applications are given to Hamiltonian system and a class of strongly indefinite elliptic system, respectively.

At the end of the section, we give some notations which will be used.

$$
\begin{aligned}
& d(A, B):=\inf \{\|x-y\| \mid x \in A, y \in B\} \\
& K(\Phi):=\left\{x \in E \mid \Phi^{\prime}(x)=0\right\} \\
& \Phi^{a}:=\{x \in E \mid \Phi(x) \leq a\}
\end{aligned}
$$

The kernel of operator $L$ is denoted by $N(L)$;

The image of operator $L$ is denoted by $R(L)$;

The space of bounded linear operators from $E$ to $F$ is denoted by $\mathbb{L}(E, F)$;

The Morse index of operator $L$ is denoted by $M^{-}(L)$;

The nullity of operator $L$ is denoted by $M^{0}(L)$.

\section{Preliminaries}

In this section, we introduce the infinite dimensional Morse theory established by Kryszewski and Szulkin. We shall give some necessary definitions and results, for more details, see [12].

Let $\left(\mathcal{G}_{n}\right)_{n=1}^{\infty}$ be a sequence of abelian groups. The asymptotic group of $\left(\mathcal{G}_{n}\right)_{n=1}^{\infty}$ is defined by

$$
\left[\left(\mathcal{G}_{n}\right)_{n=1}^{\infty}\right]:=\prod_{n=1}^{\infty} \mathcal{G}_{n} / \bigoplus_{n=1}^{\infty} \mathcal{G}_{n} .
$$

Let $\left\{E_{n}\right\}_{n=1}^{\infty}$ be a filtration of $E$, i.e. $\left\{E_{n}\right\}_{n=1}^{\infty}$ is a sequence of closed subspaces of $E, E_{n} \subseteq E_{n+1}$ and $\overline{\bigcup_{n=1}^{\infty} E_{n}}=E$. Denote $\mathcal{E}:=\left\{E_{n}, d_{n}\right\}_{n=1}^{\infty}$ and the orthogonal projector of $E$ onto $E_{n}$ by $P_{n}$, where $\left(d_{n}\right)_{n=1}^{\infty}$ is a sequence of nonnegative integers. For any integer $q$ and closed subset $(X, A)$ of $E$, we define the $q$-th $\mathcal{E}$-cohomology group of $(X, A)$ with coefficients in a fixed field $\mathcal{F}$ by

$$
H_{\mathcal{E}}^{q}(X, A):=\left[\left(H^{q+d_{n}}\left(X \cap E_{n}, A \cap E_{n}\right)\right)_{n=1}^{\infty}\right] .
$$

Here $H_{\mathcal{E}}^{*}$ satisfies all the Eilenberg-Steenrod axioms for cohomology except the dimension axiom.

A functional $\Phi \in C^{1}(E, \mathbb{R})$ is said to satisfy the (PS)* condition with respect to $\mathcal{E}$ means that, whenever a sequence $\left\{y_{j}\right\}$ is such that $y_{j} \in E_{n_{j}}$ for some $n_{j}$, $n_{j} \rightarrow \infty$, there is $M>0$ such that $\left|\Phi\left(y_{j}\right)\right|<M$ for all $j \geq 1$ and $P_{n_{j}} \nabla \Phi\left(y_{j}\right) \rightarrow 0$ as $j \rightarrow \infty$, then $\left\{y_{j}\right\}$ has a convergent subsequence. 
For an isolated critical point $p$, if $\Phi \in C^{1}(E, \mathbb{R})$ satisfies (PS)* condition, then there is an admissible pair $\left(W, W^{-}\right)$for $\Phi$ and $p$ (see page 3189 of [12]). We define the $q$-th critical group $(q \in \mathbb{Z})$ of $\Phi$ at $p$ with respect to $\mathcal{E}$ by

$$
C_{\mathcal{E}}^{q}(\Phi, p):=H_{\mathcal{E}}^{q}\left(W, W^{-}\right) .
$$

If $\Phi \in C^{1}(E, \mathbb{R})$ satisfies $(\mathrm{PS})^{*}$ condition and the critical set $K=K(\Phi)$ is compact, there is also an admissible pair $\left(W, W^{-}\right)$for $\Phi$ and $K$ with respect to $\mathcal{E}$ (see page 3193 of [12]). In particular, if there exist $a<b$ such that $K \subset$ $\operatorname{int} \Phi^{-1}([a, b])$, then $\left(\Phi^{-1}([a, b]), \Phi^{-1}(a)\right)$ is an admissible pair for $\Phi$ and $K$. We define the $q$-th critical group of $\Phi$ at infinity with respect to $\mathcal{E}$ by

$$
C_{\mathcal{E}}^{q}(\Phi, K)=H_{\mathcal{E}}^{q}\left(W, W^{-}\right)=H_{\mathcal{E}}^{q}\left(\Phi^{-1}([a, b]), \Phi^{-1}(a)\right) .
$$

Denote

$$
[\mathbb{Z}]:=\prod_{n=1}^{\infty} \mathbb{Z} / \bigoplus_{n=1}^{\infty} \mathbb{Z}, \quad\left[\mathbb{Z}_{+}\right]:=\left\{\left[\left(\xi_{n}\right)_{n=1}^{\infty}\right] \in[\mathbb{Z}]: \xi_{n} \geq 0 \text { for almost all } n\right\} .
$$

Let $(X, B)$ be a pair of closed subsets of $E$ with the property that for each $q \in \mathbb{Z}$ there is an $n(q)$ such that

$$
\operatorname{dim} H^{q+d_{n}}\left(X \cap E_{n}, B \cap E_{n}\right)<\infty, \quad \text { for all } n \geq n(q),
$$

then $\operatorname{dim}_{\mathcal{E}} H_{\mathcal{E}}^{q}(X, B):=\left[\left(\operatorname{dim} H^{q+d_{n}}\left(X \cap E_{n}, B \cap E_{n}\right)_{n=1}^{\infty}\right]\right.$ is a well-defined element of $\left[\mathbb{Z}_{+}\right]$. The sequence $\left(\operatorname{dim} H^{q+d_{n}}\left(X \cap E_{n}, B \cap E_{n}\right)_{n=1}^{\infty}\right.$ will often be constant for almost all $n$. In such a case we will write $\operatorname{dim}_{\mathcal{E}} H_{\mathcal{E}}^{q}(X, B)=[d]$, $d$ being the constant. We will say that the pair $(X, B)$ is of $\mathcal{E}$-finite type, if $\operatorname{dim}_{\mathcal{E}} H_{\mathcal{E}}^{q}(X, B)$ is well-defined and $\operatorname{dim}_{\mathcal{E}} H_{\mathcal{E}}^{q}(X, B)=[0]$ for almost all $q \in \mathbb{Z}$.

Suppose $\Phi \in C^{1}(E, \mathbb{R})$ satisfies (PS)* condition, $\left(W, W^{-}\right)$is an admissible pair for $\Phi$ and $K(\Phi)=\left\{p_{1}, \ldots p_{k}\right\}$. We call $p_{j}$ is of $\mathcal{E}$-finite type, if some(and therefore every) admissible pair for $\Phi$ and $p_{j}$ is $\mathcal{E}$-finite. If $\left(W, W^{-}\right)$and all $p_{j}$ are $\mathcal{E}$-finite, we define

$$
\begin{aligned}
M_{\mathcal{E}}^{q}\left(W, W^{-}\right) & :=\sum_{j=1}^{k} \operatorname{dim}_{\mathcal{E}} C_{\mathcal{E}}^{q}\left(\Phi, p_{j}\right), \quad q \in \mathbb{Z}, \\
\beta_{\mathcal{E}}^{q}\left(W, W^{-}\right) & :=\operatorname{dim}_{\mathcal{E}} H_{\mathcal{E}}^{q}\left(W, W^{-}\right), \quad q \in \mathbb{Z} .
\end{aligned}
$$

Moreover, in such a case we define the Morse and the Poincaré polynomials of $\left(W, W^{-}\right)$by setting

$$
M_{\mathcal{E}}\left(t, W, W^{-}\right):=\sum_{q=-\infty}^{\infty} M_{\mathcal{E}}^{q}\left(W, W^{-}\right) t^{q}, \quad P_{\mathcal{E}}\left(t, W, W^{-}\right):=\sum_{q=-\infty}^{\infty} \beta_{\mathcal{E}}^{q}\left(W, W^{-}\right) t^{q} .
$$

Here $M_{\mathcal{E}}$ and $P_{\mathcal{E}}$ are not polynomials in the usual sense, some exponents $q$ may be negative, $M_{\mathcal{E}}$ and $P_{\mathcal{E}}$ are elements of $[\mathbb{Z}]\left[t, t^{-1}\right]$. 
TheOrem 2.1 ([12]). Suppose that $\Phi \in C^{1}(E, \mathbb{R})$ satisfies $(\mathrm{PS})^{*}$ condition, $\left(W, W^{-}\right)$is an admissible pair for $\Phi$ and $K(\Phi):=\left\{p_{1}, \ldots p_{k}\right\}$. If all $p_{j}$ are $\mathcal{E}$-finite, then the pair $\left(W, W^{-}\right)$is $\mathcal{E}$-finite, and there is a polynomial

$$
Q(t)=\sum_{q=-\infty}^{\infty} a_{q} t^{q}
$$

such that $a_{q} \in\left[\mathbb{Z}_{+}\right]$, for all $q \in \mathbb{Z}$, and

$$
M_{\mathcal{E}}\left(t, W, W^{-}\right)=P_{\mathcal{E}}\left(t, W, W^{-}\right)+(1+t) Q(t)
$$

Let $E$ be a real Hilbert space with a given filtration $\mathcal{E}=\left\{E_{n}, d_{n}\right\}_{n=1}^{\infty}$.

A mapping $f: D \rightarrow E$ ( $D$ is a closed subset of $E$ ) is said to be $A$-proper (with respect to $\mathcal{E}$ ) if each bounded sequence $\left\{x_{j}\right\} \subset D$ such that $x_{j} \in E_{n_{j}} \cap D$ for some $n_{j}, n_{j} \rightarrow \infty$ and $P_{n_{j}} f\left(x_{j}\right) \rightarrow y \in E$ as $j \rightarrow \infty$, has a convergent subsequence.

Proposition 2.2 ([12]). Assume that $L \in \mathbb{L}(E, E)$ is a self-adjoint Fredholm operator of index 0 and $\mathcal{E}$ is a given filtration. $P_{n}: E \rightarrow E_{n}$ and $Q_{n}: R(L) \rightarrow$ $R(L) \cap E_{n}$ are the orthogonal projectors, then

(a) There exists an $n_{0}$ such that if $n \geq n_{0}$, then $\left.P_{n}\right|_{N(L)}: N(L) \rightarrow P_{n} N(L)$ is a linear isomorphism and $\|z\| \leq 2\left\|P_{n} z\right\|$, for all $z \in N(L)$;

(b) $E_{n}=\left(R(L) \cap E_{n}\right) \oplus P_{n} N(L)$, and the spaces $R(L) \cap E_{n}$ and $P_{n} N(L)$ are orthogonal;

(c) $P_{n}-Q_{n} \rightarrow 0$ in $\mathbb{L}(R(L)$, E) as $n \rightarrow \infty$;

(d) The sequence $\left\{R(L) \cap E_{n}\right\}_{n=1}^{\infty}$ is a filtration of $R(L)$. More precisely, for each $x \in R(L), Q_{n} x \rightarrow x$ as $n \rightarrow \infty$.

Proposition 2.3 ([12]). Let $L \in \mathbb{L}(E, E)$ be a self-adjoint operator. Then the following conditions are equivalent:

(a) $L$ is A-proper;

(b) $L$ is a Fredholm operator of index 0 and there exist $c>0, n_{0} \geq 1$ such that if $n \geq n_{0}$, then $\left\|P_{n} L x\right\| \geq c\|x\|$ for all $x \in R(L) \cap E_{n}$.

Let $L \in \mathbb{L}(E, E)$ be a self-adjoint Fredholm operator, we define the $\mathcal{E}$-Morse index $M_{\mathcal{E}}^{-}(L)$ of $L$ by

$$
M_{\mathcal{E}}^{-}(L):=\lim _{n \rightarrow \infty}\left(M^{-}\left(\left.Q_{n} L\right|_{R(L) \cap E_{n}}\right)-d_{n}\right),
$$

where $Q_{n}: R(L) \rightarrow R(L) \cap E_{n}$ is the orthogonal projector.

Proposition 2.4 ([12]). Suppose $\widetilde{L} \in \mathbb{L}(E, E)$ is a self-adjoint Fredholm operator of index 0 such that $\widetilde{L}\left(E_{n}\right) \subset E_{n}$ for almost all $n$ and $B \in \mathbb{L}(E, E)$ is 
a self-adjoint compact operator. Then $\widetilde{L}-B$ is A-proper. If $M^{-}\left(\left.\widetilde{L}\right|_{E_{n}}\right)=d_{n}+k$ for almost all $n$ and some $k \in \mathbb{Z}$, then $M_{\mathcal{E}}^{-}(L)$ is well-defined and finite.

Denote the dimension of the space $N(L)$ by $M^{0}(L)$. As usual $P_{n}: E \rightarrow E_{n}$ is the orthogonal projector from $E$ to $E_{n}$.

REMARK 2.5 ([12]). If $N(L) \subset E_{n_{0}}$ for some $n_{0}$, then when $n \geq n_{0}$,

$$
M_{\mathcal{E}}^{-}(L)=\lim _{n \rightarrow \infty}\left(M^{-}\left(\left.Q_{n} L\right|_{R(L) \cap E_{n}}\right)-d_{n}\right)=\lim _{n \rightarrow \infty}\left(M^{-}\left(\left.P_{n} L\right|_{E_{n}}\right)-d_{n}\right) .
$$

We may make use of $\mathcal{E}$-Morse index of $L$ to compute the critical group $C_{\mathcal{E}}^{*}(\Phi, p)$ at isolated critical point $p$.

TheOREm $2.6([12])$. Suppose that $\Phi \in C^{1}(E, \mathbb{R}), p$ is an isolated critical point of $\Phi$, and

$$
\Phi(x)=\Phi(p)+\frac{1}{2}\langle L(x-p), x-p\rangle-\psi(x),
$$

where $L$ is an invertible A-proper operator and $\nabla \psi(x)=o(\|x-p\|)$ as $x \rightarrow p$. If $M_{\mathcal{E}}^{-}(L)$ is well-defined and finite, then

$$
C_{\mathcal{E}}^{q}(\Phi, p)= \begin{cases}{[\mathcal{F}],} & q=M_{\mathcal{E}}^{-}(L), \\ {[0],} & q \neq M_{\mathcal{E}}^{-}(L) .\end{cases}
$$

\section{The main result}

Let $E=E^{+} \oplus E^{-} \oplus N\left(L_{\infty}\right)$ be the decomposition corresponding to the positive, negative and zero part of the spectrum of $L_{\infty}, L_{0}=\Phi^{\prime \prime}(0)$, and $\varphi_{0}(x)=$ $\Phi(x)-\left\langle L_{0} x, x\right\rangle / 2$.

We make the following assumptions:

(H1) There is a $\widetilde{L} \in \mathbb{L}(E, E)$ such that:

(1) $\widetilde{L}$ is a linear self-adjoint Fredholm operator of index 0 in $E$;

(2) $L_{\infty}=\widetilde{L}-B_{\infty}, L_{0}=\widetilde{L}-B_{0}$, where $B_{\infty}$ and $B_{0}$ are compact linear self-adjoint operators in $E$;

(3) There exists $\left\{E_{n}, d_{n}\right\}_{n=1}^{\infty}$ with $E_{n} \subset E_{n+1} \subset E, \overline{\bigcup_{n=1}^{\infty} E_{n}}=E, d_{n}$ and $k$ are nonnegative integer, such that $\widetilde{L}\left(E_{n}\right) \subset E_{n}$ and $M^{-}\left(\left.\widetilde{L}\right|_{E_{n}}\right)$ $=d_{n}+k$

(H2) $\nabla \varphi, \nabla \varphi_{0}$ are compact mappings, and $\nabla \varphi_{0}(x)=o(\|x\|)$ as $\|x\| \rightarrow 0$;

(H3) $L_{0}$ is nondegenerate, i.e. $M^{0}\left(L_{0}\right)=0$;

(H4) There exists a constant $C>0$, such that $\|\nabla \varphi(x)\|<C$ for all $x \in E$;

(H5) For a sequence $\left\{x_{j}\right\}$, let $x_{j}=x_{j}^{-}+x_{j}^{0}+x_{j}^{+}$, with $x_{j}^{ \pm} \in E^{ \pm}$and $x_{j}^{0} \in$ $N\left(L_{\infty}\right)$, if $x_{j}^{+}+x_{j}^{-}$are bounded and $\left\|x_{j}^{0}\right\| \rightarrow \infty$ as $j \rightarrow \infty$, then $\lim _{j \rightarrow \infty}\left\|\varphi^{\prime \prime}\left(x_{j}\right)\right\|=0$.

The following is our main result. 
TheOREM 3.1. Suppose (H1)-(H5) hold. If $M_{\mathcal{E}}^{-}\left(L_{0}\right)<M_{\mathcal{E}}^{-}\left(L_{\infty}\right)-1$ or $M_{\mathcal{E}}^{-}\left(L_{0}\right)>M_{\mathcal{E}}^{-}\left(L_{\infty}\right)+M^{0}\left(L_{\infty}\right)+1$, then $\Phi(x)$ has at least one nontrivial critical point.

To prove Theorem 3.1, we need some lemmas. Denote $\mathcal{E}^{\prime}=\left\{E_{n}^{\prime}, d_{n}\right\}_{n=1}^{\infty}$, where $E_{n}^{\prime}:=\left(R\left(L_{\infty}\right) \cap E_{n}\right) \oplus N\left(L_{\infty}\right)$.

Let $P_{n}^{\prime}: E \rightarrow E_{n}^{\prime}$ and $Q_{n}: R\left(L_{\infty}\right) \rightarrow R\left(L_{\infty}\right) \cap E_{n}$ be the orthogonal projectors.

Lemma 3.2. Suppose (H1) holds, then $L_{\infty}$ and $L_{0}$ are A-proper with respect to $\mathcal{E}^{\prime}$.

Proof. Since $L_{\infty}=\widetilde{L}-B_{\infty}, \widetilde{L} \in \mathbb{L}(E, E)$ is a self-adjoint Fredholm operator of index 0 and $B_{\infty} \in \mathbb{L}(E, E)$ is a self-adjoint compact operator, by Proposition 2.4, $L_{\infty}$ is $A$-proper with respect to $\mathcal{E}$.

Let $\left\{x_{j}\right\}$ be a bounded sequence such that $x_{j} \in E_{n_{j}}^{\prime}$ for some $n_{j}, n_{j} \rightarrow \infty$ and $P_{n_{j}}^{\prime} L_{\infty} \rightarrow y \in E$ as $j \rightarrow \infty$.

Suppose $x_{j}=\bar{x}_{j}+x_{j}^{0} \in\left(R\left(L_{\infty}\right) \cap E_{n_{j}}\right) \oplus N\left(L_{\infty}\right)=E_{n_{j}}^{\prime}$, let $\widehat{x}_{j}=\bar{x}_{j}+P_{n_{j}} x_{j}^{0}$. By (b) of Proposition 2.2, $E_{n_{j}}=\left(R\left(L_{\infty}\right) \cap E_{n_{j}}\right) \oplus P_{n} N\left(L_{\infty}\right)$, then $\widehat{x}_{j} \in E_{n_{j}}$ and $\left\|\widehat{x}_{j}-x_{j}\right\| \rightarrow 0$ as $j \rightarrow \infty$. Since $\left\{x_{j}\right\}$ is bounded, $\left\{\widehat{x}_{j}\right\}$ is also bounded.

It is clear that $P_{n_{j}}^{\prime} x=Q_{n_{j}} x$ for any $x \in R\left(L_{\infty}\right)$. By (c) of Proposition 2.2, $P_{n_{j}}-Q_{n_{j}} \rightarrow 0$ in $\mathbb{L}\left(R\left(L_{\infty}\right), E\right)$, we have

$$
P_{n_{j}} L_{\infty} \bar{x}_{j}-P_{n_{j}}^{\prime} L_{\infty} \bar{x}_{j}=P_{n_{j}} L_{\infty} \bar{x}_{j}-Q_{n_{j}} L_{\infty} \bar{x}_{j} \rightarrow 0 \quad \text { as } j \rightarrow \infty .
$$

Furthermore, by $\operatorname{dim} N\left(L_{\infty}\right)<\infty$, we have $\left.\left(P_{n_{j}}-I\right)\right|_{N\left(L_{\infty}\right)} \rightarrow 0$ in $\mathbb{L}\left(N\left(L_{\infty}\right), E\right)$ as $j \rightarrow \infty$, so

$$
P_{n_{j}} L_{\infty} P_{n_{j}} x_{j}^{0}=P_{n_{j}} L_{\infty}\left(P_{n_{j}}-I\right) x_{j}^{0} \rightarrow 0 \quad \text { as } j \rightarrow \infty .
$$

Hence

$$
\begin{aligned}
P_{n_{j}} L_{\infty} \widehat{x}_{j}-P_{n_{j}}^{\prime} L_{\infty} x_{j} & =P_{n_{j}} L_{\infty}\left(\bar{x}_{j}+P_{n_{j}} x_{j}^{0}\right)-P_{n_{j}}^{\prime} L_{\infty}\left(\bar{x}_{j}+x_{j}^{0}\right) \\
& =P_{n_{j}} L_{\infty} \bar{x}_{j}-P_{n_{j}}^{\prime} L_{\infty} \bar{x}_{j}+P_{n_{j}} L_{\infty} P_{n_{j}} x_{j}^{0} \rightarrow 0
\end{aligned}
$$

as $j \rightarrow \infty$. It follows from (3.1) and $P_{n_{j}}^{\prime} L_{\infty} x_{j} \rightarrow y$ that $P_{n_{j}} L_{\infty} \widehat{x}_{j} \rightarrow y$. Since $L_{\infty}$ is $A$-proper with respect to $\mathcal{E},\left\{\widehat{x}_{j}\right\}$ has a convergent subsequence. Hence $\left\{x_{j}\right\}$ also has a convergent subsequence, and $L_{\infty}$ is $A$-proper with respect to $\mathcal{E}^{\prime}$.

Similarly, we can prove $L_{0}$ is $A$-proper with respect to $\mathcal{E}^{\prime}$.

Remark 3.3. Since $L_{\infty}$ is A-proper with respect to $\mathcal{E}^{\prime}$, by Proposition 2.3, there exist $n_{0}>0$ and $C>0$ such that for $n \geq n_{0}$ and $x \in R\left(L_{\infty}\right) \cap E_{n}$, $\left\|P_{n}^{\prime} L_{\infty} x\right\| \geq C\|x\|$, thus $N\left(\left.P_{n}^{\prime} L_{\infty}\right|_{E_{n}^{\prime}}\right) \cap\left(R\left(L_{\infty}\right) \cap E_{n}\right)=\{0\}$. Note that $E_{n}^{\prime}=$ $\left(R\left(L_{\infty}\right) \cap E_{n}\right) \oplus N\left(L_{\infty}\right)$, so for $n \geq n_{0}, N\left(\left.P_{n}^{\prime} L_{\infty}\right|_{E_{n}^{\prime}}\right)=N\left(L_{\infty}\right)$. Let $E_{n}^{\prime}=$ $E_{n}^{\prime+} \oplus E_{n}^{\prime-} \oplus N\left(L_{\infty}\right)$ be the decomposition corresponding to the positive, negative and zero part of the spectrum of $\left.P_{n}^{\prime} L_{\infty}\right|_{E_{n}^{\prime}}$. By (b) of Proposition 2.3, there 
exist $n_{0}$ and a constant $C_{1}>0$ such that $\left\langle P_{n}^{\prime} L_{\infty} x, x\right\rangle \geq C_{1}\|x\|^{2}$ for $n \geq n_{0}$ and $x \in E_{n}^{\prime+},\left\langle P_{n}^{\prime} L_{\infty} x, x\right\rangle \leq-C_{1}\|x\|^{2}$ for $n \geq n_{0}$ and $x \in E_{n}^{\prime-}$.

Let $\Phi_{\rho}(x)=\Phi(x)+\chi_{\rho}\left(\left\|x^{0}\right\|^{2}\right)$, where $\chi_{\rho}(t) \in C^{2}(\mathbb{R}, \mathbb{R}), \rho>0$ and

$$
\chi_{\rho}(t)= \begin{cases}0, & t \leq \rho, \\ (t-\rho)^{4}, & t>\rho .\end{cases}
$$

Lemma 3.4. Suppose (H1), (H2) and (H4) hold, then for every $\rho>0, \Phi_{\rho}$ satisfies $(\mathrm{PS})^{*}$ condition with respect to $\mathcal{E}^{\prime}$, and for $n$ large enough $\left.\Phi_{\rho}\right|_{E_{n}^{\prime}}$ satisfies (PS) condition.

Proof. Let $\left\{x_{j}\right\}$ be a sequence with $x_{j} \in E_{n_{j}}^{\prime}$ for some $n_{j}, n_{j} \rightarrow \infty$ and $P_{n_{j}}^{\prime} \nabla \Phi_{\rho}\left(x_{j}\right) \rightarrow 0$ as $j \rightarrow \infty$.

First we show the boundedness of $\left\{x_{j}\right\}$. Let $x_{j}=x_{j}^{+}+x_{j}^{-}+x_{j}^{0} \in E_{n_{j}}^{\prime+} \oplus$ $E_{n_{j}}^{\prime-} \oplus N\left(L_{\infty}\right)$. Since $P_{n_{j}}^{\prime} \nabla \Phi_{\rho}\left(x_{j}\right) \rightarrow 0$ as $j \rightarrow \infty$, by Remark 3.3 and (H4), we have

$$
\begin{aligned}
o\left(\left\|x_{j}^{+}\right\|\right) & =\left\langle P_{n_{j}}^{\prime} \nabla \Phi_{\rho}\left(x_{j}\right), x_{j}^{+}\right\rangle \\
& =\left\langle P_{n_{j}}^{\prime} L_{\infty}\left(x_{j}\right), x_{j}^{+}\right\rangle-\left\langle P_{n_{j}}^{\prime} \nabla \varphi\left(x_{j}\right), x_{j}^{+}\right\rangle \geq C_{1}\left\|x_{j}^{+}\right\|^{2}-C_{0}\left\|x_{j}^{+}\right\|
\end{aligned}
$$

as $j \rightarrow \infty$, and

$$
\begin{aligned}
o\left(\left\|x_{j}^{-}\right\|\right) & =\left\langle P_{n_{j}}^{\prime} \nabla \Phi_{\rho}\left(x_{j}\right), x_{j}^{-}\right\rangle \\
& =\left\langle P_{n_{j}}^{\prime} L_{\infty}\left(x_{j}\right), x_{j}^{-}\right\rangle-\left\langle P_{n_{j}}^{\prime} \nabla \varphi\left(x_{j}\right), x_{j}^{-}\right\rangle \leq-C_{1}\left\|x_{j}^{-}\right\|^{2}+C_{0}\left\|x_{j}^{-}\right\|
\end{aligned}
$$

as $j \rightarrow \infty$. Hence $\left\{x_{j}^{+}\right\}$and $\left\{x_{j}^{-}\right\}$are bounded, and thus $\left\{x_{j}^{+}+x_{j}^{-}\right\}$is bounded. Moreover, by (H4),

$$
\begin{aligned}
o\left(\left\|x_{j}^{0}\right\|\right)=\left\langle P_{n_{j}}^{\prime} \nabla \Phi_{\rho}\left(x_{j}\right), x_{j}^{0}\right\rangle & =-\left\langle P_{n_{j}}^{\prime} \nabla \varphi\left(x_{j}\right), x_{j}^{0}\right\rangle+2 \chi_{\rho}^{\prime}\left(\left\|x_{j}^{0}\right\|^{2}\right)\left\|x_{j}^{0}\right\|^{2} \\
& \geq-C_{0}\left\|x_{j}^{0}\right\|+2 \chi_{\rho}^{\prime}\left(\left\|x_{j}^{0}\right\|^{2}\right)\left\|x_{j}^{0}\right\|^{2} .
\end{aligned}
$$

By the definition of $\chi_{\rho}$ and (3.3), $\left\{x_{j}^{0}\right\}$ is bounded. Hence $\left\{x_{j}\right\}$ is bounded.

Now we show that $\left\{x_{j}\right\}$ has a convergent subsequence. Since $\left\{x_{j}\right\}$ is bounded, $\nabla \varphi$ is compact and $\operatorname{dim} N\left(L_{\infty}\right)<\infty$, there exists a subsequence of $\left\{x_{j}\right\}$ (for simplicity still denote by $\left.\left\{x_{j}\right\}\right)$ and $y \in E$ such that $P_{n_{j}}^{\prime} \nabla \varphi\left(x_{j}\right)-2 \chi_{\rho}^{\prime}\left(\left\|x_{j}^{0}\right\|^{2}\right) x_{j}^{0} \rightarrow y$. Since

$$
P_{n_{j}}^{\prime} \nabla \Phi_{\rho}\left(x_{j}\right)=P_{n_{j}}^{\prime} L_{\infty}\left(x_{j}\right)-P_{n_{j}}^{\prime} \nabla \varphi\left(x_{j}\right)+2 \chi_{\rho}^{\prime}\left(\left\|x_{j}^{0}\right\|^{2}\right) x_{j}^{0} \rightarrow 0,
$$

we have $P_{n_{j}}^{\prime} L_{\infty}\left(x_{j}\right) \rightarrow y$. By Lemma $3.2, L_{\infty}$ is $A$-proper with respect to $\mathcal{E}^{\prime}$, so $\left\{x_{j}\right\}$ has a convergent subsequence. This means that $\Phi_{\rho}$ satisfies $(\mathrm{PS})^{*}$ condition with respect to $\mathcal{E}^{\prime}$.

By a similar argument we can show that for $n$ large enough, any (PS) sequence $\left\{x_{j}\right\}\left(x_{j} \in E_{n}^{\prime}\right)$ of $\left.\Phi_{\rho}\right|_{E_{n}^{\prime}}$ is bounded. Since $E_{n}^{\prime}$ is finite dimensional, $\left\{x_{j}\right\}$ has a convergent subsequence. Therefore for $n$ large enough $\left.\Phi_{\rho}\right|_{E_{n}^{\prime}}$ satisfies (PS) condition. 
Remark 3.5. Note that in Lemma 3.4 one needs not to assume $\left\{\Phi_{\rho}\left(x_{j}\right)\right\}$ to be bounded. Hence the critical point set of $\Phi_{\rho}$ is compact for every $\rho>0$.

Lemma 3.6. Suppose (H1), (H2) and (H4) hold, then $M_{\mathcal{E}^{\prime}}^{-}\left(L_{\infty}\right)=M_{\mathcal{E}}^{-}\left(L_{\infty}\right)$.

Proof. Since $N\left(L_{\infty}\right) \subset E_{n}^{\prime}$, according to Remark 2.5, we have

$$
M_{\mathcal{E}^{\prime}}^{-}\left(L_{\infty}\right)=\lim _{n \rightarrow \infty}\left(M^{-}\left(\left.P_{n}^{\prime} L_{\infty}\right|_{E_{n}^{\prime}}\right)-d_{n}\right) .
$$

On the other hand,

$$
E_{n}^{\prime}:=\left(R\left(L_{\infty}\right) \cap E_{n}\right) \oplus N\left(L_{\infty}\right) \quad \text { and }\left.\quad P_{n}^{\prime} L_{\infty}\right|_{R\left(L_{\infty}\right) \cap E_{n}}=\left.Q_{n} L_{\infty}\right|_{R\left(L_{\infty}\right) \cap E_{n}},
$$

so

$$
\begin{aligned}
M_{\mathcal{E}^{\prime}}^{-}\left(L_{\infty}\right) & =\lim _{n \rightarrow \infty}\left(M^{-}\left(\left.P_{n}^{\prime} L_{\infty}\right|_{E_{n}^{\prime}}\right)-d_{n}\right) \\
& =\lim _{n \rightarrow \infty}\left(M^{-}\left(\left.Q_{n} L_{\infty}\right|_{R\left(L_{\infty}\right) \cap E_{n}}\right)-d_{n}\right)=M_{\mathcal{E}}^{-}\left(L_{\infty}\right) .
\end{aligned}
$$

Lemma 3.7. Suppose (H1) holds. If $\Phi_{\rho}^{\prime \prime}\left(x_{0}\right)$ is invertible, then

$$
M_{\mathcal{E}^{\prime}}^{-}\left(\Phi_{\rho}^{\prime \prime}\left(x_{0}\right)\right)=M_{\mathcal{E}}^{-}\left(\Phi_{\rho}^{\prime \prime}\left(x_{0}\right)\right)
$$

Proof. Denote $L=\Phi_{\rho}^{\prime \prime}\left(x_{0}\right)$. Since $L$ is invertible, according to Remark 2.5, we have

$$
M_{\mathcal{E}^{\prime}}^{-}(L)=\lim _{n \rightarrow \infty}\left(M^{-}\left(\left.P_{n}^{\prime} L\right|_{E_{n}^{\prime}}\right)-d_{n}\right), \quad M_{\mathcal{E}}^{-}(L)=\lim _{n \rightarrow \infty}\left(M^{-}\left(\left.P_{n} L\right|_{E_{n}}\right)-d_{n}\right) .
$$

Since $\operatorname{dim} N\left(L_{\infty}\right)<\infty$, there exists $\varepsilon_{n} \rightarrow 0$ such that

$$
\left\|P_{n} z-z\right\| \leq \varepsilon_{n}\|z\|, \quad \text { for all } z \in N\left(L_{\infty}\right) .
$$

By Proposition 2.2, there exists a $n_{0}>0$ such that $P_{n}: N\left(L_{\infty}\right) \rightarrow P_{n} N\left(L_{\infty}\right)$ is a linear isomorphism when $n \geq n_{0}$. Note that

$$
E_{n}^{\prime}=\left(R\left(L_{\infty}\right) \cap E_{n}\right) \oplus N\left(L_{\infty}\right), E_{n}=\left(R\left(L_{\infty}\right) \cap E_{n}\right) \oplus P_{n} N\left(L_{\infty}\right) .
$$

When $n \geq n_{0}$, for $x=\bar{x}+x^{0} \in\left(R(L) \cap E_{n}\right) \oplus N\left(L_{\infty}\right)=E_{n}^{\prime}$, we define $H_{n}: E_{n}^{\prime} \rightarrow E_{n}$ by $H_{n}(x)=\bar{x}+P_{n} x^{0}$, then $H_{n}$ is a linear isomorphism.

Since $L$ is invertible, according to Lemma 3.2 and Proposition 2.3, there exists a $n_{1}>n_{0}$ such that $\left.P_{n}^{\prime} L\right|_{E_{n}^{\prime}}$ is also invertible when $n \geq n_{1}$. Hence, for $n \geq n_{1}$, $N\left(\left.P_{n}^{\prime} L\right|_{E_{n}^{\prime}}\right)=0$, and let $E_{n}^{\prime}=E_{n}^{\prime+} \oplus E_{n}^{\prime-}$ be the decomposition corresponding to the positive and the negative part of the spectrum of $\left.P_{n}^{\prime} L\right|_{E_{n}^{\prime}}$. For $n \geq n_{1}$ there exists a constant $C_{2}>0$ such that $\langle L x, x\rangle=\left\langle P_{n}^{\prime} L x, x\right\rangle \geq C_{2}\|x\|^{2}$ for $x \in E_{n}^{\prime+}$ and $\langle L x, x\rangle=\left\langle P_{n}^{\prime} L x, x\right\rangle \leq-C_{2}\|x\|^{2}$ for $x \in E_{n}^{\prime-}$. 
For $x \in E_{n}^{\prime-} \backslash\{0\}$, let $x=\bar{x}+x^{0} \in\left(R\left(L_{\infty}\right) \cap E_{n}\right) \oplus N\left(L_{\infty}\right)$, then by (3.4), for $n \geq n_{1}$ large enough

$$
\begin{aligned}
& \left\langle P_{n} L H_{n} x, H_{n} x\right\rangle=\left\langle L\left(\bar{x}+P_{n} x^{0}\right),\left(\bar{x}+P_{n} x^{0}\right)\right\rangle \\
& \quad=\left\langle L\left(x-x^{0}+P_{n} x^{0}\right),\left(x-x^{0}+P_{n} x^{0}\right)\right\rangle \\
& \quad=\langle L x, x\rangle+2\left\langle L x,\left(P_{n} x^{0}-x^{0}\right)\right\rangle+\left\langle L\left(P_{n} x^{0}-x^{0}\right),\left(P_{n} x^{0}-x^{0}\right)\right\rangle \\
& \quad \leq-C_{2}\|x\|^{2}+3 \varepsilon_{n}\|L\|\|x\|^{2}<0 .
\end{aligned}
$$

Since $H_{n}: E_{n}^{\prime} \rightarrow E_{n}$ is an isomorphism, then $E_{n}=H_{n} E_{n}^{\prime+} \oplus H_{n} E_{n}^{\prime-}$. Thus by (3.5), we have $\left\langle P_{n} L x, x\right\rangle<0$ for $x \in H_{n} E_{n}^{\prime-}$, this implies that

$$
M^{-}\left(\left.P_{n} L\right|_{E_{n}}\right) \geq M^{-}\left(\left.P_{n}^{\prime} L\right|_{E_{n}^{\prime}}\right) .
$$

Similarly we can prove that $\left\langle P_{n} L x, x\right\rangle>0$ for $x \in H_{n} E_{n}^{\prime+}$, and thus

$$
M^{-}\left(\left.P_{n} L\right|_{E_{n}}\right) \leq M^{-}\left(\left.P_{n}^{\prime} L\right|_{E_{n}^{\prime}}\right) .
$$

Hence $M^{-}\left(\left.P_{n}^{\prime} L\right|_{E_{n}^{\prime}}\right)=M^{-}\left(\left.P_{n} L\right|_{E_{n}}\right)$. Therefore,

$$
M_{\mathcal{E}^{\prime}}^{-}(L)=\lim _{n \rightarrow \infty}\left(M^{-}\left(\left.P_{n}^{\prime} L\right|_{E_{n}^{\prime}}\right)-d_{n}\right)=\lim _{n \rightarrow \infty}\left(M^{-}\left(\left.P_{n} L\right|_{E_{n}}\right)-d_{n}\right)=M_{\mathcal{E}}^{-}(L) .
$$

According to Theorem 2.6, (H2), (H3), Lemma 3.2 and Lemma 3.7, we have

$$
C_{\mathcal{E}^{\prime}}^{q}\left(\Phi_{\rho}, 0\right)=C_{\mathcal{E}^{\prime}}^{q}(\Phi, 0)= \begin{cases}{[\mathcal{F}],} & q=M_{\mathcal{E}}^{-}\left(L_{0}\right), \\ {[0],} & q \neq M_{\mathcal{E}}^{-}\left(L_{0}\right) .\end{cases}
$$

Lemma 3.8. Suppose (H1), (H2) and (H4) hold, then for any $\rho>0$, the critical group for $\Phi_{\rho}$ at infinity is

$$
C_{\mathcal{E}^{\prime}}^{q}\left(\Phi_{\rho}, K\left(\Phi_{\rho}\right)\right)= \begin{cases}{[\mathcal{F}],} & q=M_{\mathcal{E}}^{-}\left(L_{\infty}\right), \\ {[0],} & q \neq M_{\mathcal{E}}^{-}\left(L_{\infty}\right) .\end{cases}
$$

Proof. By Remark 3.5, $K\left(\Phi_{\rho}\right)$ is compact, so we can choose $b>0$ such that $K\left(\Phi_{\rho}\right) \subset \operatorname{int} \Phi_{\rho}^{-1}([-b, b])$. By the definition of $C_{\mathcal{E}^{\prime}}^{q}\left(\Phi_{\rho}, K\left(\Phi_{\rho}\right)\right)$, we need to compute $H_{\mathcal{E}^{\prime}}^{q}\left(\Phi_{\rho}^{-1}([-b, b]), \Phi_{\rho}^{-1}(-b)\right)$.

Consider the operator $\left.P_{n}^{\prime} L_{\infty}\right|_{E_{n}^{\prime}}: E_{n}^{\prime} \rightarrow E_{n}^{\prime}$. By Remark 3.3, for $x \in E_{n}^{\prime}$, we can set $x=x^{+}+x^{-}+x^{0} \in E_{n}^{\prime+} \oplus E_{n}^{\prime-} \oplus N\left(L_{\infty}\right)$. By (H4), for $n \geq n_{0}$ we have

$$
\left\langle P_{n}^{\prime} \nabla \Phi_{\rho}(x), x^{-}\right\rangle=\left\langle P_{n}^{\prime} L_{\infty} x, x^{-}\right\rangle-\left\langle P_{n}^{\prime} \nabla \varphi(x), x^{-}\right\rangle \leq-C_{1}\left\|x^{-}\right\|^{2}+C\left\|x^{-}\right\| .
$$

Hence there is a $R>0$ large enough such that

$$
\left\langle P_{n}^{\prime} \nabla \Phi_{\rho}(x), x^{-}\right\rangle<-a, \quad x \in E_{n}^{\prime} \backslash\left(E_{n}^{\prime} \cap U_{R}\right),
$$

where $a>0$ is a constant, $U_{R}:=\left\{x \in E \mid\left\|x^{-}\right\| \leq R\right\}$. This implies that $\left.\Phi_{\rho}\right|_{E_{n}^{\prime}}$ has no critical point in $E_{n}^{\prime} \backslash\left(E_{n}^{\prime} \cap U_{R}\right)$, and the negative gradient vector field of $\left.\Phi_{\rho}\right|_{E_{n}^{\prime}}$ points outward of $E_{n}^{\prime} \cap U_{R}$ on $\partial\left(E_{n}^{\prime} \cap U_{R}\right)$. 
By Remark 3.3 and (H4), for any $x \in E_{n}^{\prime} \cap U_{R}$, we have

$$
\begin{aligned}
\Phi_{\rho}(x) & =\frac{1}{2}\left\langle L_{\infty} x^{+}, x^{+}\right\rangle+\frac{1}{2}\left\langle L_{\infty} x^{-}, x^{-}\right\rangle-\varphi(x)+\chi_{\rho}\left(\left\|x^{0}\right\|^{2}\right) \\
& \geq \frac{1}{2} C_{1}\left\|x^{+}\right\|^{2}-\frac{1}{2}\|L\| R^{2}-C\left(\left\|x^{+}\right\|+R+\left\|x^{0}\right\|\right)+\chi_{\rho}\left(\left\|x^{0}\right\|^{2}\right),
\end{aligned}
$$

By the definition of $\chi_{\rho}$ and (3.8), we can choose $b>0$ sufficiently large such that $\Phi_{\rho}(x)>-b$ for $n \geq n_{0}$ and $x \in E_{n}^{\prime} \cap U_{R}$, therefore $\Phi_{\rho}^{-b} \cap E_{n}^{\prime} \subset E_{n}^{\prime} \backslash\left(E_{n}^{\prime} \cap U_{R}\right)$. By Lemma 3.4, we can take $n_{0}$ large enough such that $\left.\Phi_{\rho}\right|_{E_{n}^{\prime}}$ satisfies (PS) condition for $n \geq n_{0}$. Note that $\left.\Phi_{\rho}\right|_{E_{n}^{\prime}}$ has no critical points in $E_{n}^{\prime} \backslash\left(E_{n}^{\prime} \cap U_{R}\right)$ and the negative gradient vector field of $\left.\Phi_{\rho}\right|_{E_{n}^{\prime}}$ points outward of $E_{n}^{\prime} \cap U_{R}$ on $\partial\left(E_{n}^{\prime} \cap U_{R}\right)$, we can construct a deformation mapping $\gamma_{1}: E_{n}^{\prime} \backslash\left(E_{n}^{\prime} \cap U_{R}\right) \rightarrow \Phi_{\rho}^{-b} \cap E_{n}^{\prime}$ by the flow generated by $-\left.P_{n}^{\prime} \nabla \Phi_{\rho}\right|_{E_{n}^{\prime}}$.

We claim that one can choose $b$ and $n_{0}$ large enough such that for $n \geq n_{0}$, $K\left(\left.\Phi_{\rho}\right|_{E_{n}^{\prime}}\right) \subset \Phi_{\rho}^{b} \cap E_{n}^{\prime}$. Indeed, if this is not true, there is a sequence $\left\{x_{j}\right\}$ such that $x_{j} \in E_{n_{j}}^{\prime}$ for some $n_{j}, P_{n_{j}}^{\prime} \nabla \Phi_{\rho}\left(x_{j}\right)=0, n_{j} \rightarrow \infty$ and $\Phi_{\rho}\left(x_{j}\right) \rightarrow \infty$ as $j \rightarrow \infty$. Since in the proof of Lemma 3.4 we do not need to assume $\left\{\Phi_{\rho}\left(x_{j}\right)\right\}$ to be bounded, by Lemma 3.4 there exists $x_{0} \in E$ such that $\left\{x_{j}\right\}$ has a subsequence (still denoted by $\left\{x_{j}\right\}$ ) which converges to $x_{0}$, thus $\Phi_{\rho}\left(x_{j}\right) \rightarrow \Phi_{\rho}\left(x_{0}\right)$, therefore we get a contradiction. On the other hand, $\left.\Phi_{\rho}\right|_{E_{n}^{\prime}}$ satisfies (PS) condition, by deformation lemma, there is a deformation retract $\gamma_{2}: E_{n}^{\prime} \rightarrow \Phi_{\rho}^{b} \cap E_{n}^{\prime}$.

Therefore, for $n \geq n_{0}$, we have

$$
H^{q}\left(\Phi_{\rho}^{b} \cap E_{n}^{\prime}, \Phi_{\rho}^{-b} \cap E_{n}^{\prime}\right) \cong H^{q}\left(E_{n}^{\prime}, E_{n}^{\prime} \backslash\left(E_{n}^{\prime} \cap U_{R}\right)\right)= \begin{cases}\mathcal{F}, & q=M^{-}\left(\left.P_{n}^{\prime} L_{\infty}\right|_{E_{n}^{\prime}}\right), \\ 0, & q \neq M^{-}\left(\left.P_{n}^{\prime} L_{\infty}\right|_{E_{n}^{\prime}}\right) .\end{cases}
$$

By Lemma 3.6 and $M_{\mathcal{E}^{\prime}}^{-}\left(L_{\infty}\right)=\lim _{n \rightarrow \infty}\left(M^{-}\left(\left.P_{n}^{\prime} L_{\infty}\right|_{E_{n}^{\prime}}\right)-d_{n}\right)$, there exists $n_{1}>n_{0}$ such that $M_{\mathcal{E}}^{-}\left(L_{\infty}\right)=M_{\mathcal{E}^{\prime}}^{-}\left(L_{\infty}\right)=M^{-}\left(\left.P_{n}^{\prime} L_{\infty}\right|_{E_{n}^{\prime}}\right)-d_{n}$ for any $n \geq n_{1}$. Hence we have

and

$$
H^{q}\left(\Phi_{\rho}^{b} \cap E_{n}^{\prime}, \Phi_{\rho}^{-b} \cap E_{n}^{\prime}\right)= \begin{cases}\mathcal{F}, & q=M_{\mathcal{E}}^{-}\left(L_{\infty}\right)+d_{n} \\ 0, & q \neq M_{\mathcal{E}}^{-}\left(L_{\infty}\right)+d_{n}\end{cases}
$$

$$
H_{\mathcal{E}^{\prime}}^{q}\left(\Phi_{\rho}^{b}, \Phi_{\rho}^{-b}\right) \cong\left[\left(H^{q+d_{n}}\left(\Phi_{\rho}^{b} \cap E_{n}^{\prime}, \Phi_{\rho}^{-b} \cap E_{n}^{\prime}\right)\right)_{n=1}^{\infty}\right]= \begin{cases}{[\mathcal{F}],} & q=M_{\mathcal{E}}^{-}\left(L_{\infty}\right) \\ {[0],} & q \neq M_{\mathcal{E}}^{-}\left(L_{\infty}\right) .\end{cases}
$$

By excision and the definition of $C_{\mathcal{E}^{\prime}}^{q}\left(\Phi_{\rho}, K\left(\Phi_{\rho}\right)\right)$,

$$
C_{\mathcal{E}^{\prime}}^{q}\left(\Phi_{\rho}, K\left(\Phi_{\rho}\right)\right) \cong H_{\mathcal{E}^{\prime}}^{q}\left(\Phi_{\rho}^{-1}([-b, b]), \Phi_{\rho}^{-1}(-b)\right) \cong H_{\mathcal{E}^{\prime}}^{q}\left(\Phi_{\rho}^{b}, \Phi_{\rho}^{-b}\right) .
$$

Lemma 3.9. There exists a constant $M>0$ independent of $\rho$ such that for any $x \in K\left(\Phi_{\rho}\right)$ with $x=x^{+}+x^{-}+x^{0} \in E^{+} \oplus E^{-} \oplus N\left(L_{\infty}\right)$, we have $\left\|x^{+}+x^{-}\right\|<M$. 
Proof. For $x \in K\left(\Phi_{\rho}\right)$ with $x=x^{+}+x^{-}+x^{0} \in E^{+} \oplus E^{-} \oplus N\left(L_{\infty}\right)$, by (H4), one has

$$
\begin{aligned}
& 0=\left\langle\nabla \Phi_{\rho}(x), x^{+}\right\rangle=\left\langle L_{\infty} x, x^{+}\right\rangle-\left\langle\nabla \varphi(x), x^{+}\right\rangle \geq C\left\|x^{+}\right\|^{2}-C_{0}\left\|x^{+}\right\|, \\
& 0=\left\langle\nabla \Phi_{\rho}(x), x^{-}\right\rangle=\left\langle L_{\infty} x, x^{-}\right\rangle-\left\langle\nabla \varphi(x), x^{-}\right\rangle \leq-C\left\|x^{-}\right\|^{2}+C_{0}\left\|x^{-}\right\|,
\end{aligned}
$$

where $C_{0}$ and $C$ are constants independent of $\rho$. So there exist constants $M_{1}, M_{2}>0$ independent of $\rho>0$ such that for $x \in K\left(\Phi_{\rho}\right)$, we have $\left\|x^{+}\right\|<M_{1}$, $\left\|x^{-}\right\|<M_{2}$. Let $M=M_{1}+M_{2}$, we have $\left\|x^{+}+x^{-}\right\|<M$.

Proof of Theorem 3.1. For $x \in E$, let $x=x^{+}+x^{-}+x^{0} \in E^{+} \oplus E^{-} \oplus$ $N\left(L_{\infty}\right)$. If $\Phi$ has no nontrivial critical point, then $\Phi_{\rho}$ has no nontrivial critical point in set $E_{\rho}:=\left\{x \in E \mid\left\|x^{0}\right\|^{2} \leq \rho\right\}$. Since $M_{\mathcal{E}}^{-}\left(L_{0}\right) \neq M_{\mathcal{E}}^{-}\left(L_{\infty}\right)$, the critical set $K\left(\Phi_{\rho}\right) \backslash\{0\}$ is not empty. According to Remark 3.5, $K\left(\Phi_{\rho}\right) \backslash\{0\}$ is compact, so $d:=d\left(K\left(\Phi_{\rho}\right), E_{\rho}\right)>0$. Let $N_{\delta}(A):=\{x \in E \mid d(x, A)<\delta\}$. By the MarinoProdi perturbation technique [20], for any $\varepsilon>0,0<\tau<\min \{1, d / 3\}$, there exists a $C^{2}$ functional $I_{\rho}$ such that

(a) $\left\|\Phi_{\rho}-I_{\rho}\right\|_{C^{2}}<\varepsilon$;

(b) $\Phi_{\rho}(x)=I_{\rho}(x), x \in E \backslash N_{2 \tau}\left(K\left(\Phi_{\rho}\right)\right)$;

(c) $\Phi_{\rho}^{\prime \prime}(x)=I_{\rho}^{\prime \prime}(x), x \in N_{\tau}\left(K\left(\Phi_{\rho}\right)\right), K\left(I_{\rho}\right) \subset N_{\tau}\left(K\left(\Phi_{\rho}\right)\right)$, and the critical points of $I_{\rho}$ are all nondegenerate.

By (b), (3.6) and Lemma 3.8 we have

$$
\begin{gathered}
C_{\mathcal{E}^{\prime}}^{q}\left(I_{\rho}, K\left(I_{\rho}\right)\right)=C_{\mathcal{E}^{\prime}}^{q}\left(\Phi_{\rho}, K\left(\Phi_{\rho}\right)\right)= \begin{cases}{[\mathcal{F}],} & q=M_{\mathcal{E}}^{-}\left(L_{\infty}\right), \\
{[0],} & q \neq M_{\mathcal{E}}^{-}\left(L_{\infty}\right),\end{cases} \\
C_{\mathcal{E}^{\prime}}^{q}\left(I_{\rho}, 0\right)=C_{\mathcal{E}^{\prime}}^{q}\left(\Phi_{\rho}, 0\right)= \begin{cases}{[\mathcal{F}],} & q=M_{\mathcal{E}}^{-}\left(L_{0}\right), \\
{[0],} & q \neq M_{\mathcal{E}}^{-}\left(L_{0}\right) .\end{cases}
\end{gathered}
$$

We claim that: there exists a constant $\beta>0$ independent of $\rho$ such that if $x \in$ $K\left(I_{\rho}\right)$ satisfies $M_{\mathcal{E}}^{-}\left(I_{\rho}^{\prime \prime}(x)\right)<M_{\mathcal{E}}^{-}\left(L_{\infty}\right)$ or $M_{\mathcal{E}}^{-}\left(I_{\rho}^{\prime \prime}(x)\right)>M_{\mathcal{E}}^{-}\left(L_{\infty}\right)+M^{0}\left(L_{\infty}\right)$, then $\left\|x^{0}\right\| \leq \beta$.

If the claim is not true, then there exists a sequence $\left\{x_{i}\right\}$ such that $x_{i} \in$ $K\left(I_{\rho_{i}}\right), \rho_{i}>0, M_{\mathcal{E}}^{-}\left(I_{\rho_{i}}^{\prime \prime}\left(x_{i}\right)\right)<M_{\mathcal{E}}^{-}\left(L_{\infty}\right)$ or $M_{\mathcal{E}}^{-}\left(I_{\rho_{i}}^{\prime \prime}\left(x_{i}\right)\right)>M_{\mathcal{E}}^{-}\left(L_{\infty}\right)+M^{0}\left(L_{\infty}\right)$, and $\left\|x_{i}^{0}\right\| \rightarrow \infty$ as $i \rightarrow \infty$

Since $K\left(I_{\rho_{i}}\right) \subset N_{\tau}\left(K\left(\Phi_{\rho_{i}}\right)\right)$, there exists $x_{i}^{\prime} \in K\left(\Phi_{\rho_{i}}\right)$ such that $\left\|x_{i}-x_{i}^{\prime}\right\|<$ $\tau<1$. By Lemma 3.9, $\left\|x_{i}^{\prime+}+x_{i}^{\prime-}\right\|<M$. Hence $\left\|x_{i}^{+}+x_{i}^{-}\right\|<M+1$. On the other hand $\left\|x_{i}^{0}\right\| \rightarrow \infty$ as $i \rightarrow \infty$, so by (H5), $\mid \varphi^{\prime \prime}\left(x_{i}\right) \| \rightarrow 0$ as $i \rightarrow \infty$. Hence there exists $i_{0}>0$ such that for any $i \geq i_{0}$, we have $\left\|\varphi^{\prime \prime}\left(x_{i}\right)\right\|<C_{1} / 2$, where $C_{1}$ is given in Remark 3.3. 
For $n \geq n_{0}, y^{-} \in E_{n}^{\prime-} \backslash\{0\}, y^{+} \in E_{n}^{\prime+} \backslash\{0\}$, by Remark 3.3 and (c), we get

$$
\begin{aligned}
\left\langle P_{n}^{\prime} I_{\rho}^{\prime \prime}\left(x_{i}\right) y^{-}, y^{-}\right\rangle & =\left\langle P_{n}^{\prime} \Phi_{\rho}^{\prime \prime}\left(x_{i}\right) y^{-}, y^{-}\right\rangle \\
& =\left\langle P_{n}^{\prime} L_{\infty} y^{-}, y^{-}\right\rangle-\left\langle P_{n}^{\prime} \varphi^{\prime \prime}\left(x_{i}\right) y^{-}, y^{-}\right\rangle \\
& \leq-C_{1}\left\|y^{-}\right\|^{2}+\left\|\varphi^{\prime \prime}\left(x_{i}\right)\right\|\left\|y^{-}\right\|^{2}<0, \\
\left\langle P_{n}^{\prime} I_{\rho}^{\prime \prime}\left(x_{i}\right) y^{+}, y^{+}\right\rangle & =\left\langle P_{n}^{\prime} \Phi_{\rho}^{\prime \prime}\left(x_{i}\right) y^{+}, y^{+}\right\rangle \\
& =\left\langle P_{n}^{\prime} L_{\infty} y^{+}, y^{+}\right\rangle-\left\langle P_{n}^{\prime} \varphi^{\prime \prime}\left(x_{i}\right) y^{+}, y^{+}\right\rangle \\
& \geq C_{1}\left\|y^{+}\right\|^{2}-\left\|\varphi^{\prime \prime}\left(x_{i}\right)\right\|\left\|y^{+}\right\|^{2}>0 .
\end{aligned}
$$

Hence for $n \geq n_{0}$ and $i \geq i_{0}$,

$$
\begin{aligned}
& M^{-}\left(\left.P_{n}^{\prime} I_{\rho}^{\prime \prime}\left(x_{i}\right)\right|_{E_{n}^{\prime}}\right) \geq M^{-}\left(\left.P_{n}^{\prime} L_{\infty}\right|_{E_{n}^{\prime}}\right), \\
& M^{-}\left(\left.P_{n}^{\prime} I_{\rho}^{\prime \prime}\left(x_{i}\right)\right|_{E_{n}^{\prime}}\right) \leq M^{-}\left(\left.P_{n}^{\prime} L_{\infty}\right|_{E_{n}^{\prime}}\right)+M^{0}\left(L_{\infty}\right) .
\end{aligned}
$$

Since the critical points $x_{i}$ are all nondegenerate, by Lemma 3.7, (3.11), (3.12) and (c) for $i \geq i_{0}$, we have

$$
\begin{aligned}
M_{\mathcal{E}}^{-}\left(I_{\rho}^{\prime \prime}\left(x_{i}\right)\right) & =M_{\mathcal{E}^{\prime}}^{-}\left(I_{\rho}^{\prime \prime}\left(x_{i}\right)\right)=\lim _{n \rightarrow \infty}\left(M^{-}\left(\left.P_{n}^{\prime} I_{\rho}^{\prime \prime}\left(x_{i}\right)\right|_{E_{n}^{\prime}}\right)-d_{n}\right) \\
& \geq \lim _{n \rightarrow \infty}\left(M^{-}\left(\left.P_{n}^{\prime} L_{\infty}\right|_{E_{n}^{\prime}}\right)-d_{n}\right)=M_{\mathcal{E}^{\prime}}^{-}\left(L_{\infty}\right)=M_{\mathcal{E}}^{-}\left(L_{\infty}\right), \\
M_{\mathcal{E}}^{-}\left(I_{\rho}^{\prime \prime}\left(x_{i}\right)\right) & =M_{\mathcal{E}^{\prime}}^{-}\left(I_{\rho}^{\prime \prime}\left(x_{i}\right)\right)=\lim _{n \rightarrow \infty}\left(M^{-}\left(\left.P_{n}^{\prime} I_{\rho}^{\prime \prime}\left(x_{i}\right)\right|_{E_{n}^{\prime}}\right)-d_{n}\right) \\
& \leq \lim _{n \rightarrow \infty}\left(M^{-}\left(\left.P_{n}^{\prime} L_{\infty}\right|_{E_{n}^{\prime}}\right)-d_{n}\right)+M^{0}\left(L_{\infty}\right) \\
& =M_{\mathcal{E}^{\prime}}^{-}\left(L_{\infty}\right)+M^{0}\left(L_{\infty}\right)=M_{\mathcal{E}}^{-}\left(L_{\infty}\right)+M^{0}\left(L_{\infty}\right) .
\end{aligned}
$$

This leads to a contradiction. The claim is proved.

Take $\rho=\beta^{2}+1$, then $I_{\rho}$ has no nontrivial critical point in the set $E_{\rho}$, so for $x \in K\left(I_{\rho}\right) \backslash\{0\}$, we have $\left\|x^{0}\right\|>\beta$. By the above claim, for any $x \in K\left(I_{\rho}\right) \backslash\{0\}$,

$$
M_{\mathcal{E}}^{-}\left(L_{\infty}\right) \leq M_{\mathcal{E}}^{-}\left(I_{\rho}^{\prime \prime}(x)\right) \leq M_{\mathcal{E}}^{-}\left(L_{\infty}\right)+M^{0}\left(L_{\infty}\right) .
$$

Since the critical set $K\left(I_{\rho}\right)$ of $I_{\rho}$ is compact, and the elements of $K\left(I_{\rho}\right)$ are all nondegenerate, $K\left(I_{\rho}\right)$ is a finite set. Assume that $K\left(I_{\rho}\right) \backslash\{0\}=\left\{x_{1}, \ldots, x_{n}\right\}$, and denote $m_{\mathcal{E}}^{-}\left(x_{i}\right)=M_{\mathcal{E}}^{-}\left(I_{\rho}^{\prime \prime}\left(x_{i}\right)\right)$. From (3.9), (3.10), Theorems 2.6 and 2.1 we know that

$$
t^{M_{\mathcal{E}}^{-}\left(L_{0}\right)}+\sum_{i=1}^{n} t^{m_{\overline{\mathcal{E}}}^{-}\left(x_{i}\right)}=t^{M_{\mathcal{E}}^{-}\left(L_{\infty}\right)}+(1+t) Q(t) .
$$

Since the left-hand side of (3.14) contains the exponent $M_{\mathcal{E}}^{-}\left(L_{0}\right)$ and $M_{\mathcal{E}}^{-}\left(L_{0}\right) \neq$ $M_{\mathcal{\varepsilon}}^{-}\left(L_{\infty}\right)$. Therefore $Q(t)$ must have a nonzero term with exponent $M_{\mathcal{E}}^{-}\left(L_{0}\right)$ or $M_{\mathcal{E}}^{-}\left(L_{0}\right)-1$, and it follows that there is a nonzero term with exponent $M_{\mathcal{E}}^{-}\left(L_{0}\right)+1$ or $M_{\mathcal{E}}^{-}\left(L_{0}\right)-1$ on the left-hand side. Hence there exists $x_{i} \in K\left(I_{\rho}\right)$ such that $m_{\mathcal{E}}^{-}\left(x_{i}\right)=M_{\mathcal{\varepsilon}}^{-}\left(L_{0}\right)-1$ or $m_{\mathcal{E}}^{-}\left(x_{i}\right)=M_{\mathcal{E}}^{-}\left(L_{0}\right)+1$. So according to the condition $M_{\mathcal{E}}^{-}\left(L_{0}\right)<M_{\mathcal{E}}^{-}\left(L_{\infty}\right)-1$ or $M_{\mathcal{E}}^{-}\left(L_{0}\right)>M_{\mathcal{E}}^{-}\left(L_{\infty}\right)+M^{0}\left(L_{\infty}\right)+1$, we 
have $m_{\mathcal{E}}^{-}\left(x_{i}\right)<M_{\mathcal{E}}^{-}\left(L_{\infty}\right)$ or $m_{\mathcal{E}}^{-}\left(x_{i}\right)>M_{\mathcal{E}}^{-}\left(L_{\infty}\right)+M^{0}\left(L_{\infty}\right)$. This contradicts to (3.13), so $\Phi$ has at least one nontrivial critical point.

\section{Application to Hamiltonian systems}

Consider the following Hamiltonian systems

$$
\dot{z}=J H_{z}(z, t),
$$

where $J=\left(\begin{array}{cc}0 & -I \\ I & 0\end{array}\right)$ is the standard symplectic matrix.

First we introduce the following assumptions:

(A1) $H \in C^{2}\left(\mathbb{R}^{2 N} \times \mathbb{R}\right)$ is $2 \pi$-periodic in $t$;

(A2) $H(z, t)=\frac{1}{2} A(t) z \cdot z+G(z, t)$, where $A(t)$ is a symmetric $2 N \times 2 N$ matrix with $2 \pi$-periodic entries, and $\left|G_{z}(z, t)\right| \leq C$ for some $C>0$ and all $(z, t) \in \mathbb{R}^{2 N} \times \mathbb{R}$

(A3) $H(z, t)=\frac{1}{2} A_{0}(t) z \cdot z+G_{0}(z, t)$, where $A_{0}(t)$ is a symmetric $2 N \times 2 N$ matrix with $2 \pi$-periodic entries, and $\left(G_{0}\right)_{z}(z, t)=o(|z|)$ uniformly in $t$ as $|z| \rightarrow 0$;

(A4) The equation $\dot{z}=J A_{0}(t) z$ has no nontrivial $2 \pi$-periodic solution;

(A5) $\left\|G_{z z}(z, t)\right\| \rightarrow 0$ uniformly in $t$ as $|z| \rightarrow \infty$.

Let

$$
\begin{aligned}
E=\left\{z(t) \mid z(t)=a_{0}+\sum_{k=1}^{\infty} a_{k} \cos k t+b_{k} \sin k t,\right. & a_{0}, a_{k}, b_{k} \in \mathbb{R}^{2 N}, \\
& \left.\sum_{k=1}^{\infty} k\left(\left|a_{k}\right|^{2}+\left|b_{k}\right|^{2}\right)<\infty\right\} .
\end{aligned}
$$

Then $E$ is a Hilbert space with inner product $\langle\cdot, \cdot\rangle$ defined by

$$
\left\langle z, z^{\prime}\right\rangle=2 \pi a_{0} \cdot a_{0}^{\prime}+\pi \sum_{k=1}^{\infty} k\left(a_{k} \cdot a_{k}^{\prime}+b_{k} \cdot b_{k}^{\prime}\right) .
$$

Let

$$
\Phi=\frac{1}{2} \int_{0}^{2 \pi}(-J \dot{z} \cdot z) d t-\int_{0}^{2 \pi} H(z, t) d t .
$$

By (A2) and (A5), $\left\|H_{z z}(z, t)\right\| \leq C^{\prime}\left(1+|z|^{s}\right)$ for some $C^{\prime}>0, s \in(0, \infty)$ and all $(z, t) \in \mathbb{R}^{2 N} \times \mathbb{R}$, then it is known [18] that $\Phi \in C^{2}(E, \mathbb{R})$ and $z(t)$ is a $2 \pi$-periodic solution of (4.1) if and only if it is a critical point of $\Phi$.

Let $\widetilde{L}, B_{\infty}$ and $B_{0}$ be the linear operators from $E$ to $E$ defined by

$$
\begin{aligned}
\left\langle\widetilde{L} z, z^{\prime}\right\rangle & :=\int_{0}^{2 \pi}\left(-J \dot{z} \cdot z^{\prime}\right) d t, \quad \text { forall } z, z^{\prime} \in E, \\
\left\langle B_{\infty} z, z^{\prime}\right\rangle & :=\int_{0}^{2 \pi}\left(A(t) z \cdot z^{\prime}\right) d t, \quad \text { for all } z, z^{\prime} \in E,
\end{aligned}
$$




$$
\left\langle B_{0} z, z^{\prime}\right\rangle:=\int_{0}^{2 \pi}\left(A_{0}(t) z \cdot z^{\prime}\right) d t, \quad \text { for all } z, z^{\prime} \in E .
$$

Then $\widetilde{L}$ is a self-adjoint Fredholm operator of index $0, B_{\infty}$ and $B_{0}$ are compact self-adjoint operators.

Let

$$
\varphi(z)=\int_{0}^{2 \pi} G(z, t) d t, \varphi_{0}(z)=\int_{0}^{2 \pi} G_{0}(z, t) d t .
$$

Then $\nabla \varphi$ and $\nabla \varphi_{0}$ are compact mappings, satisfy $\nabla \varphi(x)=o(\|x\|)$ as $\|x\| \rightarrow \infty$ and $\nabla \varphi_{0}(x)=o(\|x\|)$ as $\|x\| \rightarrow 0$.

Denote $L_{\infty}=\widetilde{L}-B_{\infty}, L_{0}=\widetilde{L}-B_{0}$, we can rewrite $\Phi$ by

$$
\Phi=\frac{1}{2}\left\langle L_{\infty} z, z\right\rangle-\varphi(z)=\frac{1}{2}\left\langle L_{0} z, z\right\rangle-\varphi_{0}(z) .
$$

Let

$$
E_{n}:=\left\{z \in E \mid z(t)=a_{0}+\sum_{k=1}^{n} a_{k} \cos k t+b_{k} \sin k t, a_{0}, a_{k}, b_{k} \in \mathbb{R}^{2 N}\right\},
$$

then $\left\{E_{n}\right\}_{n=1}^{\infty}$ is a filtration of $E$. Set $d_{n}:=N(2 n+1)$ and $\mathcal{E}:=\left\{E_{n}, d_{n}\right\}_{n=1}^{\infty}$, then it is easy to see that $\widetilde{L}\left(E_{n}\right) \subset E_{n}$ and $M^{-}\left(\left.\widetilde{L}\right|_{E_{n}}\right)=2 n N=d_{n}-N$. Hence $\Phi$ satisfies conditions (H1), (H2).

It follows from (A4) that $\Phi$ satisfies the condition (H3).

It follows from (A2) that

$$
|\langle\nabla \varphi(z), w\rangle|=\left|\int_{0}^{2 \pi} G_{z}(z, t) w d t\right| \leq \int_{0}^{2 \pi}\left|G_{z}(z, t)\|w \mid d t \leq C\| w \|,\right.
$$

for all $z, w \in E$ for some $C>0$, so $\|\nabla \varphi(z)\| \leq C, \forall z \in E$. Hence $\Phi$ satisfies the condition (H4).

In what following we show that $\Phi$ satisfies the condition $(H 5)$, the idea is similar to that in [3].

LEMma 4.1. Suppose (A1), (A2) and (A5) hold, then the functional $\Phi$ satisfies ( $\mathrm{H} 5)$.

Proof. Assume that the sequence $\left\{x_{j}\right\}$ satisfies that $\left\{x_{j}^{+}+x_{j}^{-}\right\}$is bounded and $\left\|x_{j}^{0}\right\| \rightarrow \infty$ as $j \rightarrow \infty$, we will show that $\left\|\varphi^{\prime \prime}\left(x_{j}\right)\right\| \rightarrow 0$ as $j \rightarrow \infty$.

First, since $\left\{x_{j}^{+}+x_{j}^{-}\right\}$is bounded and $E \hookrightarrow L^{2}\left([0,2 \pi], \mathbb{R}^{2 N}\right)$ is compact embedding, the sequence $\left\{x_{j}^{+}+x_{j}^{-}\right\}$contains in a compact subset of $L^{2}\left([0,2 \pi], \mathbb{R}^{2 N}\right)$. Since $x_{j}^{0} \in N\left(L_{\infty}\right), x_{j}^{0}$ satisfies the equation

$$
\dot{x}_{j}^{0}=J A(t) x_{j}^{0},
$$

then we obtain

$$
x_{j}^{0}(t)=x_{j}^{0}\left(t_{0}\right)+\int_{t_{0}}^{t} J A(s) x_{j}^{0}(s) d s,
$$


thus

$$
\left|x_{j}^{0}(t)\right| \leq\left|x_{j}^{0}\left(t_{0}\right)\right|+\int_{t_{0}}^{t}|J A(s)|\left|x_{j}^{0}(s)\right| d s \leq\left|x_{j}^{0}\left(t_{0}\right)\right|+C \int_{0}^{2 \pi}\left|x_{j}^{0}(s)\right| d s,
$$

where $C>0$ is a constant. It follows from Gronwall inequality that

$$
\max \left\{\left|x_{j}^{0}(t)\right| \mid t \in[0,2 \pi]\right\} \leq C^{\prime} \min \left\{\mid x_{j}^{0}(t) \| t \in[0,2 \pi]\right\} .
$$

Since $\left\|x_{j}^{0}\right\| \rightarrow \infty$ as $j \rightarrow \infty$ and $\operatorname{dim} N\left(L_{\infty}\right)<\infty$, according to the equivalence of the norm between finite dimension spaces, we have $\left\|x_{j}^{0}\right\|_{C^{0}} \rightarrow \infty$ as $j \rightarrow \infty$, that is $\max \left\{\left|x_{j}^{0}(t)\right| \mid t \in[0,2 \pi]\right\} \rightarrow \infty$ as $j \rightarrow \infty$. By (4.2), one has

$$
\min \left\{\left|x_{j}^{0}(t)\right| \mid t \in[0,2 \pi]\right\} \rightarrow \infty \quad \text { as } j \rightarrow \infty .
$$

Denote $\bar{x}_{j}=x_{j}^{+}+x_{j}^{-}$. We claim that: for any $\varepsilon>0$ there exists $M>0$ such that

$$
\operatorname{mes}\left\{t \mid \bar{x}_{j}(t) \geq M\right\}<\varepsilon \text {. }
$$

If the claim is not true, then there exists $\varepsilon_{0}>0$ such that there is a subsequence of $\left\{x_{j}\right\}$ (still denoted by $\left\{x_{j}\right\}$ ) such that $\operatorname{mes}\left\{t \mid \bar{x}_{j}(t) \geq M_{j}\right\}>\varepsilon_{0}$, where $M_{j} \rightarrow \infty$ as $j \rightarrow \infty$. Since the sequence $\left\{\bar{x}_{j}\right\}$ contains in a compact subset of $L^{2}\left([0,2 \pi], \mathbb{R}^{2 N}\right)$, there exists $x_{0} \in E$ such that in $L^{2}\left([0,2 \pi], \mathbb{R}^{2 N}\right)$ there is a subsequence of $\left\{\bar{x}_{j}\right\}$ (still denoted by $\left\{\bar{x}_{j}\right\}$ ), converges to $x_{0}$, thus, we have

$$
\int_{0}^{2 \pi}\left|\bar{x}_{j}(t)\right|^{2} d t \rightarrow \int_{0}^{2 \pi}\left|x_{0}(t)\right|^{2} d t<\infty
$$

This contradicts to

$$
\int_{0}^{2 \pi}\left|\bar{x}_{j}(t)\right|^{2} d t \geq M_{j}^{2} \varepsilon_{0} \rightarrow \infty, \quad j \rightarrow \infty .
$$

The claim is proved.

For any $\varepsilon>0$, by the above claim and (A5), there exist $M>0$ and $X>0$ such that $\operatorname{mes}\left\{t \mid \bar{x}_{j}(t) \geq M, t \in[0,2 \pi]\right\}<\varepsilon$, and $\left\|G_{z z}(z, t)\right\|<\varepsilon$ for $|z|>X$. Denote $Y=\left\{t \mid \bar{x}_{j}(t) \leq M, t \in[0,2 \pi]\right\}$ and since $\min \left\{\left|x_{j}^{0}(t)\right| \mid t \in[0,2 \pi]\right\} \rightarrow \infty$ as $j \rightarrow \infty$, there exists $j_{0}>0$ such that

$$
\left|x_{j}(t)\right|=\left|\bar{x}_{j}(t)+x_{j}^{0}(t)\right| \geq\left|x_{j}^{0}(t)\right|-M>X
$$

for $t \in Y$ and $j \geq j_{0}$. Moreover, by (A5), there exists $M_{0}>0$ such that $\sup \left\{\left\|G_{z z}(z, t)\right\| \mid(z, t) \in \mathbb{R}^{2 N} \times \mathbb{R}\right\} \leq M_{0}$. Hence for $j \geq j_{0}$, we have

$$
\begin{aligned}
\int_{0}^{2 \pi}\left\|G_{z z}\left(x_{j}(t), t\right)\right\|^{2} d t= & \int_{Y}\left\|G_{z z}\left(x_{j}(t), t\right)\right\|^{2} d t \\
& +\int_{[0,2 \pi] \backslash Y}\left\|G_{z z}\left(x_{j}(t), t\right)\right\|^{2} d t \leq 2 \pi \varepsilon^{2}+M_{0}^{2} \varepsilon .
\end{aligned}
$$


By Hölder inequality and Sobolev inequality, if $j \geq j_{0}$ we have

$$
\begin{aligned}
\left|\left\langle\varphi^{\prime \prime}\left(x_{j}\right) v, v\right\rangle\right| & =\left|\int_{0}^{2 \pi} G_{z z}\left(x_{j}(t), t\right) v \cdot v d t\right| \leq \int_{0}^{2 \pi}\left\|G_{z z}\left(x_{j}(t), t\right)\right\||v|^{2} d t \\
& \leq\left(\int_{0}^{2 \pi}\left\|G_{z z}\left(x_{j}(t), t\right)\right\|^{2} d t\right)^{1 / 2}\left(\int_{0}^{2 \pi}|v|^{4} d t\right)^{1 / 2} \\
& \leq\left(2 \pi \varepsilon^{2}+M_{0}^{2} \varepsilon\right)^{1 / 2}\|v\|_{L^{4}\left([0,2 \pi], \mathbb{R}^{2 N}\right)}^{2} \leq C^{\prime}\left(2 \pi \varepsilon^{2}+M_{0}^{2} \varepsilon\right)^{1 / 2}\|v\|_{E}^{2}
\end{aligned}
$$

where $C^{\prime}>0$ is a constant. Hence $\left\|\varphi^{\prime \prime}\left(x_{j}\right)\right\| \rightarrow 0$ as $j \rightarrow \infty$, $\Phi$ satisfies the condition ( $\mathrm{H} 5)$.

Note that $L_{\infty}$ and $L_{0}$ are decided by $A(t)$ and $A_{0}(t)$ respectively, we can denote $j^{-}(A):=M_{\mathcal{E}}^{-}\left(L_{\infty}\right), j^{0}(A):=M^{0}\left(L_{\infty}\right), j^{-}\left(A_{0}\right):=M_{\mathcal{E}}^{-}\left(L_{0}\right), j^{0}\left(A_{0}\right):=$ $M^{0}\left(L_{0}\right)$. According to Theorem 3.1 we have the following result.

THEOREM 4.2. Suppose that (4.1) satisfies conditions (A1)-(A5), and $j^{-}\left(A_{0}\right)$ $<j^{-}(A)-1$ or $j^{-}\left(A_{0}\right)>j^{-}(A)+j^{0}(A)+1$, then (4.1) has at least one nontrivial $2 \pi$-periodic solution.

REMARK 4.3. There are many results about the existence of nontrivial solutions of Hamiltonian systems resonant at infinity, some used the LandersmanLazer type conditions, which implies that the global compactness $\left((\mathrm{PS})^{*}\right.$ condition) of the functional is guaranteed, see [12], [22], some used strong resonance condition, which implies that the functional satisfies the (PS)* condition apart from some exceptional levels, see [5], [19]. Here we use a different condition, which is similar as in [21] for second order elliptic equation. Under this condition, the (PS)* condition may fail at any level.

\section{Application to elliptic system}

Consider the following strongly indefinite elliptic system

$$
\begin{cases}-\triangle u=F_{v}(x, u, v) & \text { in } \Omega, \\ -\triangle v=F_{u}(x, u, v) & \text { in } \Omega, \\ u=v=0 & \text { on } \partial \Omega,\end{cases}
$$

where $\Omega \subset \mathbb{R}^{N}$ is a bounded domain with smooth boundary. Problem (5.1) has been studied in [6] for subquadratic $F$, in [7], [11] for superquadratic $F$. Here we are interested in the asymptotically quadratic case and introduce the following assumptions:

(B1) $F(x, u, v) \in C^{2}\left(\bar{\Omega} \times \mathbb{R}^{2}, \mathbb{R}\right)$;

(B2) $F(x, u, v)=\frac{1}{2} a(x) u^{2}+b(x) u v+\frac{1}{2} c(x) v^{2}+G(x, u, v)$, where $a(x), b(x), c(x) \in C(\bar{\Omega}, \mathbb{R})$, and there exists $C>0$ such that $\left|G_{u}(x, u, v)\right|+\left|G_{v}(x, u, v)\right|<C$, for all $(x, u, v) \in \Omega \times \mathbb{R}^{2}$;

(B3) $F(x, u, v)=\frac{1}{2} a_{0}(x) u^{2}+b_{0}(x) u v+\frac{1}{2} c_{0}(x) v^{2}+G_{0}(x, u, v)$, 
where $a_{0}(x), b_{0}(x), c_{0}(x) \in C(\bar{\Omega}, \mathbb{R})$, and $\left|\left(G_{0}\right)_{u}(x, u, v)\right|+\left|\left(G_{0}\right)_{v}(x, u, v)\right|=$ $o(|u|+|v|)$ uniformly in $x$ as $|u|+|v| \rightarrow 0$;

(B4) The equation

$$
\begin{cases}-\triangle u=b_{0}(x) u+c_{0}(x) v & \text { in } \Omega, \\ -\triangle v=a_{0}(x) u+b_{0}(x) v & \text { in } \Omega, \\ u=v=0 & \text { on } \partial \Omega\end{cases}
$$

has no nonzero solution;

(B5) $\left\|D^{2} G(x, u, v)\right\| \rightarrow 0$ uniformly in $x$ as $|u|+|v| \rightarrow \infty$.

Let $H_{0}^{1}(\Omega)$ be the usual Sobolev space and set $E:=H_{0}^{1}(\Omega) \times H_{0}^{1}(\Omega)$. Then $E$ is a Hilbert space with inner product given by

$$
\left\langle(u, v),\left(u^{\prime}, v^{\prime}\right)\right\rangle=\int_{\Omega}\left(\nabla u \cdot \nabla u^{\prime}+\nabla v \cdot \nabla v^{\prime}\right) d x, \quad \text { for all }(u, v),\left(u^{\prime}, v^{\prime}\right) \in E .
$$

It follows from (B1), (B2) and (B5) that the functional $\Phi: E \rightarrow \mathbb{R}$ defined by

$$
\Phi(u, v)=\int_{\Omega} \nabla u \cdot \nabla v d x-\int_{\Omega} F(x, u, v) d x
$$

is of class $C^{2}(E, \mathbb{R})$ and critical points of $\Phi$ correspond to weak solutions of (5.1) [18].

Let $0<\lambda_{1}<\lambda_{2} \leq \ldots$ be the eigenvalues of the operator $-\triangle$ in $H_{0}^{1}(\Omega)$ and let $\left(e_{n}\right)_{n=1}^{\infty}$ be the corresponding orthonormal basis of eigenfunctions.

Set $E_{n}:=\operatorname{span}\left\{\left(e_{i}, 0\right),\left(0, e_{j}\right): 1 \leq i, j \leq n\right\}, \mathcal{E}=\left\{E_{n}, n\right\}_{n=1}^{\infty}$.

For $(u, v),\left(u^{\prime}, v^{\prime}\right) \in E$, we define the linear operators $\widetilde{L}, B_{\infty}$ and $B_{0}$ by

$$
\begin{aligned}
\left\langle\widetilde{L}(u, v),\left(u^{\prime}, v^{\prime}\right)\right\rangle & :=\int_{\Omega}\left(\nabla v \cdot \nabla u^{\prime}+\nabla u \cdot \nabla v^{\prime}\right) d x, \\
\left\langle B_{\infty}(u, v),\left(u^{\prime}, v^{\prime}\right)\right\rangle & :=\int_{\Omega}\left(a(x) u u^{\prime}+b(x) u^{\prime} v+b(x) u v^{\prime}+c(x) v v^{\prime}\right) d x, \\
\left\langle B_{0}(u, v),\left(u^{\prime}, v^{\prime}\right)\right\rangle & :=\int_{\Omega}\left(a_{0}(x) u u^{\prime}+b_{0}(x) u^{\prime} v+b_{0}(x) u v^{\prime}+c_{0}(x) v v^{\prime}\right) d x .
\end{aligned}
$$

Then $\widetilde{L}$ is a self-adjoint Fredholm operator of index $0, \widetilde{L}\left(E_{n}\right) \subset E_{n}, M^{-}\left(\left.\widetilde{L}\right|_{E_{n}}\right)=n$. $B_{\infty}$ and $B_{0}$ are compact self-adjoint operator. Let

$$
\varphi(u, v)=\int_{\Omega} G(x, u, v) d x, \quad \varphi_{0}(u, v)=\int_{\Omega} G_{0}(x, u, v) d x,
$$

then $\nabla \varphi$ and $\nabla \varphi_{0}$ are compact mappings. By (B2) and (B3), $\nabla \varphi(u, v)=$ $o(\|(u, v)\|)$ as $\|(u, v)\| \rightarrow \infty$ and $\nabla \varphi_{0}(u, v)=o(\|(u, v)\|)$ as $\|(u, v)\| \rightarrow 0$. Denote $L_{\infty}=\widetilde{L}-B_{\infty}, L_{0}=\widetilde{L}-B_{0}$, we can rewrite $\Phi$ by

$$
\Phi=\frac{1}{2}\left\langle L_{\infty}(u, v),(u, v)\right\rangle-\varphi(u, v)=\frac{1}{2}\left\langle L_{0}(u, v),(u, v)\right\rangle-\varphi_{0}(u, v) .
$$

Then $\Phi$ satisfies conditions (H1) and (H2).

By (B4), $\Phi$ satisfies the condition (H3). 
By (B2), for any $(u, v),\left(u^{\prime}, v^{\prime}\right) \in E$, one has

$$
\begin{aligned}
\left|\left\langle\nabla \varphi(u, v),\left(u^{\prime}, v^{\prime}\right)\right\rangle\right| & =\left|\int_{\Omega}\left(G_{u}(x, u, v) u+G_{v}(x, u, v) v\right) d x\right| \\
& \leq \int_{\Omega}\left(\left|G_{u}(x, u, v)\right|+\left|G_{v}(x, u, v)\right|\right)(|u|+|v|) d x \\
& \leq C \int_{\Omega}(|u|+|v|) d x \leq C^{\prime}\|(u, v)\|
\end{aligned}
$$

where $C^{\prime}>0$ is a constant. So $\|\nabla \varphi(u, v)\| \leq C^{\prime}$, for all $(u, v) \in E$. Hence $\Phi$ satisfies the condition (H4).

To prove $\Phi$ satisfies the condition (H5), we should reference the proposition from $[14]$.

Proposition 5.1 ([14]). Let $\Omega$ be an open domain in $\mathbb{R}^{N}$ and $M \in L_{\text {loc }}^{\infty}(\Omega, \Lambda)$, where $\Lambda$ is the linear space of $m \times m$ real symmetric matrices. If $u \in\left(H_{\mathrm{loc}}^{1}(\Omega)\right)^{m}$ satisfies the inequality $|\triangle u| \leq|M u|$ and $u$ vanishes on a subset $W$ of $\Omega$ with positive measure, then $u$ is identically zero in $\Omega$.

Now we prove that $\Phi$ satisfies the condition (H5).

Lemma 5.2. Suppose (B1), (B2) and (B5) hold, then $\Phi$ satisfies the condition ( $\mathrm{H} 5)$.

Proof. Let the sequence $\left\{\left(u_{j}, v_{j}\right)\right\}$ satisfies that $\left\{\left(u_{j}^{+}, v_{j}^{+}\right)+\left(u_{j}^{-}, v_{j}^{-}\right)\right\}$is bounded and $\left\|\left(u_{j}^{0}, v_{j}^{0}\right)\right\| \rightarrow \infty$ as $j \rightarrow \infty$. We will show that $\left\|\varphi^{\prime \prime}\left(u_{j}, v_{j}\right)\right\| \rightarrow 0$ as $j \rightarrow \infty$.

First, since $\left\{\left(u_{j}^{+}, v_{j}^{+}\right)+\left(u_{j}^{-}, v_{j}^{-}\right)\right\}$is bounded and $E \hookrightarrow L^{2}(\Omega) \times L^{2}(\Omega)$ is a compact imbedding mapping, the sequence $\left\{\left(u_{j}^{+}, v_{j}^{+}\right)+\left(u_{j}^{-}, v_{j}^{-}\right)\right\}$should contain in a compact subset of $L^{2}(\Omega) \times L^{2}(\Omega)$. Since $\left(u_{j}^{0}, v_{j}^{0}\right) \in N\left(L_{\infty}\right),\left(u_{j}^{0}, v_{j}^{0}\right)$ should satisfies the following equation

$$
\begin{cases}-\triangle u_{j}^{0}=b(x) u_{j}^{0}+c(x) v_{j}^{0} & \text { in } \Omega, \\ -\triangle v_{j}^{0}=a(x) u_{j}^{0}+b(x) v_{j}^{0} & \text { in } \Omega \\ u_{j}^{0}=v_{j}^{0}=0 & \text { on } \partial \Omega .\end{cases}
$$

Let $\left(\widetilde{u}_{j}, \widetilde{v}_{j}\right)=\left(u_{j}^{0}, v_{j}^{0}\right) /\left\|\left(u_{j}^{0}, v_{j}^{0}\right)\right\|$, then $\left(\widetilde{u}_{j}, \widetilde{v}_{j}\right)$ satisfies the equation

$$
\begin{cases}-\triangle \widetilde{u}_{j}=b(x) \widetilde{u}_{j}+c(x) \widetilde{v}_{j} & \text { in } \Omega, \\ -\triangle \widetilde{v}_{j}=a(x) \widetilde{u}_{j}+b(x) \widetilde{v}_{j} & \text { in } \Omega, \\ \widetilde{u}_{j}=\widetilde{v}_{j}=0 & \text { on } \partial \Omega .\end{cases}
$$

Since $\left\|\left(\widetilde{u}_{j}, \widetilde{v}_{j}\right)\right\|=1$, there exists $(\widetilde{u}, \widetilde{v}) \in E$ such that $\left(\widetilde{u}_{j}, \widetilde{v}_{j}\right)$ weakly convergence in $E$ and strongly convergence in $L^{2}(\Omega) \times L^{2}(\Omega)$ to $(\widetilde{u}, \widetilde{v}) \in E$, and $(\widetilde{u}, \widetilde{v})$ satisfies 
the following equation

$$
\begin{cases}-\triangle \widetilde{u}=b(x) \widetilde{u}+c(x) \widetilde{v} & \text { in } \Omega, \\ -\triangle \widetilde{v}=a(x) \widetilde{u}+b(x) \widetilde{v} & \text { in } \Omega, \\ \widetilde{u}=\widetilde{v}=0 & \text { on } \partial \Omega .\end{cases}
$$

Since $(\widetilde{u}, \widetilde{v}) \neq 0$, according to Proposition $5.1(\widetilde{u}, \widetilde{v})$ is not equal to zero almost everywhere in $\Omega$. Thus by $\left\|\left(u_{j}^{0}, v_{j}^{0}\right)\right\| \rightarrow \infty$ as $j \rightarrow \infty,\left|u_{j}^{0}\right|+\left|v_{j}^{0}\right| \rightarrow \infty$ almost everywhere in $\Omega$. Using this fact and (B5) we can follows similarly to the proof of Lemma 4.1 and obtain

$$
\int_{\Omega}\left\|D^{2} G(x, u, v)\right\|^{N / 2} d x \rightarrow 0 \quad \text { as } j \rightarrow \infty .
$$

Now by Hölder inequality and Sobolev inequality, one has

$$
\begin{aligned}
\left|\left\langle\varphi^{\prime \prime}\left(u_{j}, v_{j}\right) v, v\right\rangle\right| & =\left|\int_{\Omega} D^{2} G(x, u, v) v \cdot v d x\right| \\
& \leq\left(\int_{\Omega}\left\|D^{2} G(x, u, v)\right\|^{N / 2}\right)^{2 / N}\left(\int_{\Omega}|v|^{2 N /(N-2)}\right)^{(N-2) / N} \\
& \leq\left(\int_{\Omega}\left\|D^{2} G(x, u, v)\right\|^{N / 2}\right)^{2 / N} C^{\prime}\|v\|^{2}, \quad \text { for all } v \in E .
\end{aligned}
$$

Hence $\lim _{j \rightarrow \infty}\left\|\varphi^{\prime \prime}\left(u_{j}, v_{j}\right)\right\|=0$, then we proved $\Phi$ satisfies the condition (H5).

Note that $L_{\infty}$ is determined by $a(x), b(x)$ and $c(x), L_{0}$ is determined by $a_{0}(x), b_{0}(x)$ and $c_{0}(x)$, we can denote

$$
i_{\infty}^{-}:=M_{\mathcal{E}}^{-}\left(L_{\infty}\right), \quad i_{\infty}^{0}:=M^{0}\left(L_{\infty}\right), \quad i_{0}^{-}:=M_{\mathcal{E}}^{-}\left(L_{0}\right), \quad i_{0}^{0}:=M^{0}\left(L_{0}\right)
$$

According to Theorem 3.1 we have the following result.

THEOREM 5.3. Suppose that (5.1) satisfies the conditions (B1)-(B5), and $i_{0}^{-}<i_{\infty}^{-}-1$ or $i_{0}^{-}>i_{\infty}^{-}+i_{\infty}^{0}+1$, then (5.1) has at least one nontrivial weak solution.

REMARK 5.4. In [12], the authors studied the existence of nontrivial solutions for elliptic system (5.1) under the Landesman-Lazer type condition, which implies that the functional satisfies the (PS)* condition. However, under the condition of Theorem 5.3, the (PS)* condition may fail at any level.

Acknowledgements. The authors are grateful to the referees for their careful reading of the paper and helpful suggestions. 


\section{REFERENCES}

[1] A. AbBondandolo, A new cohomology for the Morse theory of strongly indefinite functionals on Hilbert spaces, Topol. Methods Nonlinear Anal. 9 (1997), 325-382.

[2] Morse theory for asymptotcally linear Hamiltonian systems, Nonlinear Anal. 39 (2000), 997-1049.

[3] P. Bartolo, V. Benci and D. Fortunato, Abstract critical point theorem and applications to nonlinear problems with "strong" resonance at infinity, Nonlinear Anal. 7 (1983), 981-1012.

[4] K.-C. Chang, Infinite Dimensional Morse Theory and Multiple Solution Problem, Birkhäuser, Boston, 1993.

[5] K.C. Chang, J.Q. LiU And M.J. LiU, Nontrivial periodic solutions for strong resonance Hamiltonian systems, Ann. Inst. H. Poincaré Anal. Non Linéaire 14 (1997), 103-117.

[6] D.G. Costa And C.A. Magalhães, A variational method to subquadratic perturbations of elliptic systems, J. Differential Equations 111 (1994), 103-122.

[7] D.G. De Figueiredo And P. Felmer, On superqadratic elliptic systems, Trans. Amer. Math. Soc. 111 (1994), 99-116.

[8] Y.X. GUO, Morse theury for strongly indefinite functional and its applications, Doctoral thesis, Institute of Mathematics, Peking University, 1999.

[9]_, Nontrivial periodic solutions for asymptotically linear Hamiltonian systems with resonance, J. Differential Equations 175 (2001), 71-87.

[10] Nontrivial solutions for resonant noncooperative elliptic systems, Comm. Pure Appl. Math. 53 (2000), 1335-1349.

[11] J. Hulshof AND R. VAN DER VORST, Differential systems with strongly indefinite variational structure, J. Func. Anal. 114 (1993), 32-58.

[12] W. Kryszewski and A. Szulkin, An infinite dimensional Morse theory with applications, Trans. Amer. Math. Soc. 349 (1997), 3181-3234.

[13] E. Landesman And A.C. LAZER, Nonlinear perturbations of linear eigenvalues problem at resonance, J. Math. Mech. 19 (1970), 609-623.

[14] Z.L. LiU, J.B. Su AND Z.-Q. WANG, Solutions of elliptic problems with nonlinearities of linear growth, Calc. Var. Partial Differential Equations 35 (2009), 463-480.

[15] A. Masiello and L. Pisani, Asymptotically linear elliptic problems at resonance, Ann. Math. Pura Appl. 4 (1996), 1-13.

[16] J. Mawhin And M. Willem, Critical Point Theory and Hamiltonian Systems, SpringerVerlag, New York, 1989.

[17] A. Pomponio, An asymptotically linear non-cooperative elliptic system with lack of compactness, Proc. Roy. Soc. Londondon A 459 (2003), 2265-2279.

[18] P.H. Rabinowitz, Minimax methods in critical point theory with applications to differential equations, AMS Conf. Ser. Math., vol. 65, 1986.

[19] A. Salvatore, Periodic solutions of asymptotically linear systems without symmetry, Rend. Sem. Math. Univ. 74 (1985), 147-161.

[20] M. Solimini, Morse index estimates in minimax theores, Manus. Math. 63 (1989), 421453.

[21] J.B. Su AND Z.L. LIU, Bounded resonance problem for elliptic equations, Discrete Contin. Dyn. Syst 19 (2007), 431-445.

[22] A. Szulkin, Cohomology and Morse theory for strong indefinite functionals, Math. Z. 209 (1992), 375-418.

[23] A. SzUlKin And W.M. Zou, Infinite dimensional cohomology groups and periodic solutions of asymptotically linear Hamiltonian systems, J. Differentail Equations 174 (2001), 369-391. 
[24] P.A. ZEng, J.Q. LiU And Y.X. GuO, Computations of critical groups and applications to asymptotically linear wave equation and beam equation, J. Math. Anal. Appl. 300 (2004), 102-128.

[25] W. M. Zou, Computations of the cohomology groups with applications to asymptotically linear beam equtions and noncooperative elliptic systems, Comm. Partial Differential Equations 27 (2002), 115-147.

GuangGang LiU

Department of Mathematics Science

Liaocheng University

Liaocheng 252000, P.R. CHINA

and

College of Mathematics

Jilin University

Changchun 130012, P.R. CHINA

E-mail address: $\operatorname{lgg} 112 @ 163 . c o m$

ShaOyun SHI

College of Mathematics

Jilin University

Changchun 130012, P.R. CHINA

and

Key Laboratory of Symbolic Computation

and Knowledge Engineering

of Ministry of Education

Jilin University

ChangChun University 130012, P.R. China

E-mail address: shisy@mail.jlu.edu.cn

YuCHENG WeI

College of Mathematics

Jilin University

Changchun 130012, P.R. CHINA

and

Department of Mathematics

Hechi University

Yizhou 546300, P.R.CHINA

E-mail address: ychengwei@126.com

TMNA : Volume $43-2014-\mathrm{N}^{\circ} 2$ 